\title{
ON RAMIFICATION THEORY IN THE IMPERFECT RESIDUE FIELD CASE
}

\author{
IGOR ZHUKOV
}

June 10, 2001

Let $K$ be a complete discrete valuation field with the residue field $\bar{K}$, char $\bar{K}=$ $p>0$. If $\bar{K}$ is a perfect field, there exists a beautiful theory of ramification in algebraic extensions of $K$. Given a finite Galois extension $L / K$ with the Galois group $G$, one can introduce a canonical filtration $\left(G_{i}\right)$ in $G$ with quite a natural behavior with respect to subextensions in $L / K$. Namely, if $H$ is a normal subgroup in $G$, one has $H_{i}=G_{i} \cap H$ and $(G / H)^{j}=\left(G^{j} H\right) / H$. In the last relation we used upper numbering of ramification subgroups $G^{j}=G_{\psi(j)}$, where the Hasse-Herbrand function $\psi=\psi_{L / K}$ can be easily calculated in terms of orders of $G_{i}$. Next, this "upper" filtration of $G$ is compatible with class field theory. In particular, if $L / K$ is abelian and the residue fields are finite (or quasi-finite), then $\theta\left(U_{j}\right)=G^{j}$ for all $j=0,1, \ldots$, where $\theta: K^{*} \rightarrow G$ is the reciprocity map, and $\left(U_{j}\right)$ is the filtration in $K^{*}$ determined by the valuation. A comprehensive exposition of all these facts is given, e. g., in [S, Ch. IV, Ch.XV].

However, if $\bar{K}$ is not perfect, there exists no reasonable theory of upper numbering of ramification subgroups. The "lower" ramification subgroups can still be defined, however, the ramification filtration in the group $G$ does not determine that in $G / H$. (Examples were given, e. g., in $[\mathrm{L}, \mathrm{H}]$.)

In the present article we treat the class of fields $K$ with $\left[\bar{K}: \bar{K}^{p}\right]=p$. (In particular, this holds for a two-dimensional local field $K$.) In the case char $K=p$, we work with a relative situation $K / k$, when a complete subfield $k$ in $K$ with a perfect residue field is supposed to be fixed. (In the mixed characteristic case, i. e., when char $K=0$, a subfield $k$ can be chosen in a canonical way.) For a Galois extension $L / K$ we introduce a new lower filtration on $\operatorname{Gal}(L / K)$ indexed by a special linearly ordered set $\mathbb{I}$ (see $\S 1$ ). Then a Hasse-Herbrand function $\Psi_{L / K}: \mathbb{I} \rightarrow \mathbb{I}$ can be defined with all the usual properties. Therefore, a theory of upper ramification groups, as well as the ramification theory of infinite extensions, can be developed.

If we consider abelian extensions of exponent $p$, the ramification filtration determines a dual filtration on the additive (resp. multiplicative) group of $K$ via Artin-Schreier (resp. Kummer) duality. In the case $e_{K / k}=1$, this dual filtration is described explicitly in $\S 2$.

In $\S 3$, we consider a field $K$ with a discrete valuation of rank two. (Main examples are provided by 2-dimensional local or local-global fields.) We introduce a new index

Supported by a grant of LMS, a research grant of Nottingham University (ROF2/039) and grants of RFBR (projects 97-01-00058-a and 00-01-00140)

Typeset by $\mathcal{A}_{\mathcal{M}} \mathcal{S}-\mathrm{T}_{\mathrm{E}} \mathrm{X}$ 
set $\mathbb{I}_{2} \supset \mathbb{I}$ in this case. This yields finer lower and upper filtrations on Galois groups of extensions of $K$.

In the remaining part of the article we deal with an equal characteristic twodimensional local field $K$. We introduce some filtration on the group $K_{2}^{\text {top }} K$, which is other than the filtration induced by the valuation. Our filtration is indexed by $\mathbb{I}_{2}$, and it has a better behavior with respect to the norm map than the usual filtration. Finally, we prove that the reciprocity map of two-dimensional local class field theory (see $[\mathrm{P}, \mathrm{F} 1]$ ) identifies this filtration with the ramification filtration of $\S 3$.

The ramification filtration constructed in this paper for 2-dimensional local fields has been generalized to $n$-dimensional local fields by V. A. Abrashkin (see [A1, A2]). He announced a theorem which is a local version of Grothendieck anabelian conjecture and which is stated in terms of this filtration.

The author expresses his gratitude to the University of Nottingham for hospitality, very friendly and stimulating atmosphere. I am deeply grateful to Prof. I. B. Fesenko for many valuable discussions and for his generous help during the author's stay in Nottingham.

The final version of this paper has been written during the author's stay at IHES (Bures-sur-Yvette) in 2001. I would like to thank this institute for its remarkable hospitatlity.

\section{$\S 0$. DEFINITIONS, NOTATION AND PRELIMINARY FACTS}

\subsection{General notation.}

The letter $p$ always denotes a prime number. This is the characteristic of residue fields of all discrete valuation fields under consideration; $v_{p}(a)$ is the $p$-adic exponent of a rational or $p$-adic number $a$.

Let $K$ be a field with a discrete valuation of rank 1 . Then

- $v_{K}$ denotes the (normalized) valuation of $K$ as well as its prolongation onto the algebraic closure of $K$ (which is unique provided that $K$ is complete);

- $\mathcal{O}_{K}$ is the valuation ring;

- $\mathfrak{M}_{K}$ is the maximal ideal of $\mathcal{O}_{K}$;

$\bullet \bar{K}=\mathcal{O}_{K} / \mathfrak{M}_{K}$

- $U_{K}=\mathcal{O}_{K}^{*}$;

- $U_{K}(m)=\left\{u \in \mathcal{O}_{K} \mid v_{K}(u-1) \geq m\right\}, m \geq 1$;

- $\mathcal{R}_{K}$ consists of Teichmüller representatives of elements of the maximal perfect subfield in $\bar{K}$;

A finite extension $L / K$ is said to be ferociously ramified if $[L: K]=[\bar{L}: \bar{K}]_{\text {insep }}$. The group $\mathbb{Q}^{2}=\mathbb{Q} \times \mathbb{Q}$ is linearly ordered as follows:

$$
\left(a_{1}, a_{2}\right)<\left(b_{1}, b_{2}\right) \Longleftrightarrow\left\{\begin{array}{l}
\text { either } a_{2}<b_{2} \\
\text { or } a_{2}=b_{2} \text { and } a_{1}<b_{1} ;
\end{array}\right.
$$

- $\mathbb{Q}_{+}=\{i \in \mathbb{Q} \mid i>0\}$;

- $\mathbb{Q}_{+}^{2}=\mathbb{Q} \times \mathbb{Q}_{+}$.

0.2. Elimination of wild ramification. (See $[\mathrm{E}, \mathrm{H}, \mathrm{Z1}, \mathrm{KZ}]$.)

Let $K$ be a complete discrete valuation field of any characteristic with the residue field $\bar{K}$ of characteristic $p>0$. 
If $K$ is of characteristic 0 , denote by $k$ the set of all $x \in K$ which are algebraic over the fraction field $k_{0}$ of $W(F)$, where $F=\bigcap \bar{K}^{p^{i}}$. Obviously, $k$ is a complete subfield of $K$ with perfect residue field, and it is maximal with respect to this property.

Next, if char $K=p$, we fix a base subfield $B$ in $K$, which is complete with respect to the valuation of $K$ and has $\mathbb{F}_{p}$ as a residue field. (The possible base subfields are exactly all the $\mathbb{F}_{p}((\tau))$, where $v_{K}(\tau)>0$.) In this case we denote by $k_{0}$ the completion of $B\left(\mathcal{R}_{K}\right)$, and by $k$ the algebraic closure of $k_{0}$ in $K$.

In both cases $k$ is said to be the constant subfield of $K$. We denote by $v_{0}$ the valuation $K^{*} \rightarrow \mathbb{Q}$ which is equivalent to $v_{K}$ and such that $\left.v_{0}\right|_{k_{0}}=v_{k_{0}}$. Denote $e_{K}=e_{K / k_{0}}=v_{0}(\pi)^{-1}$, where $\pi$ is any prime of $K$.

An extension $L / K$ is said to be constant if $L=l K$ where $l$ is a certain algebraic extension of $k$. Obviously, in this case $l$ is the constant subfield of $L$. Notice that finite separable constant extensions of $K$ are exactly $K(a) / K$ where $a$ is algebraic and separable over $k$. An extension is said to be almost constant if it lies in a compositum of a constant extension and an unramified one. Equivalently, $L / K$ is almost constant if $K^{\prime} L / K^{\prime}$ is a constant extension for some unramified extension $K^{\prime} / K$. Notice the following properties.

1. The compositum of two almost constant extensions is almost constant. Therefore, one can consider the maximal almost constant subextension in a given finite extension.

2. Any intermediate extension in an almost constant extension $L / K$ is also almost constant. Indeed, let $K \subset K^{\prime} \subset L^{\prime} \subset L$. We have $L \subset C U$, where $C / K$ is a constant extension, $U / K$ is an unramified one. Then $L^{\prime} \subset\left(C K^{\prime}\right)\left(U K^{\prime}\right), C K^{\prime} / K^{\prime}$ is a constant extension, $U K^{\prime} / K^{\prime}$ is an unramified one.

3. If $L / K$ and $M / L$ are almost constant, then $M / K$ is almost constant.

Indeed, $L U / U$ and $M U^{\prime} / U^{\prime}$ are constant for some unramified extensions $U / K$ and $U^{\prime} / L$. Let $U^{\prime \prime}$ be the inertia subfield in $U^{\prime} / K$. Then $U^{\prime}=U^{\prime \prime} L$, whence $M U^{\prime}=M U^{\prime \prime}$. Now $U U^{\prime \prime} / K$ is unramified, $L U U^{\prime \prime} / U U^{\prime \prime}$ and $M U U^{\prime \prime} / L U U^{\prime \prime}$ are constant, whence $M / K$ is almost constant.

4. Any tamely ramified extension is almost constant.

We give the name infernal to the opposite type of extensions. Namely, a finite extension $L / K$ is said to be infernal if the only almost constant subextension in $L / K$ is $K$ itself. The following two properties are proved in the subsection $\mathbf{1 . 2}$ of $[\mathrm{KZ}]$.

1. If $L / K$ is infernal, then any intermediate extension in $L / K$ is infernal.

2. If $L / K$ is infernal, then $K^{\prime} L / K^{\prime}$ is also infernal for any almost constant extension $K^{\prime} / K$.

We say that $K$ is standard if $e_{K / k}=1$. It is obvious that any unramified or constant or ferociously ramified finite extension of a standard field is a standard field as well.

Further, $K$ is said to be almost standard if there exists an unramified extension $L / K$ such that $L$ is standard. It is easy to prove that an almost constant or ferociously ramified finite extension of an almost standard field is almost standard.

The following statement is a particular case of Theorem 3.2.1 in [KZ]. However, a simpler proof in this case can be given.

Proposition 0.2.1. Let $K$ be a complete discrete valuation field, char $K=p, k$ the constant subfield of $K, L / K$ an infernal extension. Then there exists a finite 
purely inseparable extension $l / k$ such that $l K$ is almost standard, and $l L / l K$ is ferociously ramified.

Proof. Notice that $K$ is a finite extension of a standard field $K_{0}$. For example, one can write $K=H((\pi))$ and take $K_{0}=H\left(\left(\pi_{0}\right)\right)$, where $\pi_{0}$ is a prime in the base subfield. Let $K_{1} / K_{0}$ be the maximal almost constant subextension in $K / K_{0}$. Then $K / K_{1}$ is infernal, and $K_{1}$ is almost standard. It is sufficient to prove both assertions for $K_{2} L / K_{2} K$, where $K_{2} / K_{1}$ is any given unramified extension. Therefore, we may assume without loss of generality that $K_{1}$ is standard.

Now it is sufficient to check that $l L / l K_{1}$ is ferociously ramified for a certain purely inseparable $l / k_{1}$, where $k_{1}$ is the constant subfield in $K_{1}$. Using induction on $\left[L: K_{1}\right]$, we reduce this assertion to the case $\left[L: K_{1}\right]=p$.

First, let $L / K_{1}$ be inseparable; then $L=K_{1}(x), x^{p}=a \in K_{1}$. The fact that $L / K_{1}$ is infernal implies that $\left\{v_{K_{1}}\left(a-b^{p}-c\right) \mid b \in K_{1}, c \in k_{1}\right\}$ is bounded from above. Then one can write $a=a_{0}+b^{p}+c$ with the maximal possible $v_{K_{1}}\left(a_{0}\right)<\infty$. Let $l=k_{1}\left(\pi_{l}\right)$, where $\pi_{l}^{p}$ is a prime in $k_{1}$. Then $l L=l K_{1}\left(\sqrt[p]{a_{0}}\right)$ is ferociously ramified over $l K_{1}$.

Next, assume that $L / K_{1}$ is separable and normal. Then $L=K_{1}(x), x^{p}-x=$ $a \in K_{1}$. Let $\pi$ be a prime in $k_{1}$. Then we may assume $K_{1}=H_{1}((\pi))$.

Write $a=a_{0}+\wp(b)+c$ with $b \in K_{1}, c \in k_{1}$ with maximal possible $v\left(a_{0}\right)$. Suppose $v\left(a_{0}\right) \geq 0$. Then $L \subset K_{1}\left(x_{0}, x_{c}\right), x_{0}^{p}-x_{0}=a_{0}, x_{c}^{p}-x_{c}=c$, whence $L / K_{1}$ is almost constant, a contradiction. Therefore, $v\left(a_{0}\right)=-n<0$.

Let $s=s_{L / K_{1}}=\min \left\{r \mid \overline{\pi^{n} a_{0}} \in\left(\bar{K}_{1}\right)^{p^{r}}\right\}$. We have $s<\infty$ by the maximality of $v\left(a_{0}\right)$. Now we use induction on $s$.

If $s_{L / K_{1}}=0$, then already $L\left(\pi^{\prime}\right) / K_{1}\left(\pi^{\prime}\right)$ is ferociously ramified, where $\left(\pi^{\prime}\right)^{p}=\pi$. If $s \geq 1$, it is clear that $(p, n)=1$. Denote $L^{\prime}=L\left(\pi^{\prime}\right), K^{\prime}=K_{1}\left(\pi^{\prime}\right),\left(\pi^{\prime}\right)^{p}=\pi$. We distinguish two cases.

1. $a_{0}$ can be written in the form

$$
a_{0}=\sum_{i=-n}^{[-n / p]} b_{i}^{p} \pi^{i}+a_{1}
$$

where $b_{i} \in H, v_{K}\left(a_{1}\right)>-n / p$. Then $a_{0} \equiv b_{m}\left(\pi^{\prime}\right)^{m}+\ldots \bmod \wp\left(K^{\prime}\right)$, where $m=-n / p$, dots denote terms of higher order, and we obtain $s_{L^{\prime} / K^{\prime}}=s_{L / K_{1}}-1$.

2. $a_{0}$ cannot be written in this form. Then we see immediately that $s_{L^{\prime} / K^{\prime}}=0$.

In both cases one applies the assumption of induction to $L^{\prime} / K^{\prime}$.

It remains to consider the case when $L / K_{1}$ is of degree $p$, separable, but not normal. Let $L_{1} / K_{1}$ be the normal closure of $L / K_{1}, K_{2} / K_{1}$ the maximal tamely ramified subextension in $L_{1} / K_{1}$. Then $\left[L_{1}: K_{2}\right]=p, L_{1} / K_{2}$ is normal, and $K_{2}$ is almost standard. We can apply the already considered case to $U L_{1} / U$, where $U$ is a suitable unramified extension of $K_{2}$.

Proposition 0.2.2. (Epp [E]) Let $L / K$ be a finite extension of complete discrete valuation fields, $k$ the constant subfield of $K$. Then there exists a finite extension $l / k$ such that $e_{l L / l K}=1$.

Proof. Let char $K=p$. Let $K^{\prime} / K$ be the maximal almost constant subextension in $L / K, k^{\prime}$ the constant subfield in $K^{\prime}$. Then $K^{\prime}$ is almost standard, and $L / K^{\prime}$ is infernal. There exists $l_{1} / k$ such that $l_{1} K^{\prime} / l_{1} K$ is unramified. By Proposition 0.2.1, 
there exists $l_{2} / k^{\prime}$ such that $l_{2} L / l_{2} K^{\prime}$ is ferociously ramified. Let $l=l_{1} l_{2}$. Then $l K^{\prime} / l K$ is unramified, $l L / l K^{\prime}$ is ferociously ramified, whence $e(l L / l K)=1$.

In the case char $K=0$ the argument is essentially the same. Instead of purely inseparable extensions $l / k$ one can take cyclic extensions with sufficiently big ramification jumps. For a detailed proof, see [Z1, KZ].

Corollary. Let $K$ be a complete discrete valuation field, $k$ the constant subfield of $K$. Then there exists a finite extension $l / k$ such that $l K$ is standard.

0.3. Two-dimensional local fields. (See [HLF, MZ1, MZ2].)

We shall freely use the terminology and notation from [HLF, MZ1]. In particular, a two-dimensional local field is a field $K$ which is complete with respect to a discrete valuation $v$, and such that the residue field $\bar{K}$ of $K$ is a complete discretely valued field with perfect residue field. Throughout this article we assume that $\bar{K}$ is of characteristic $p$.

A system of local parameters in $K$ is any $(t, \pi)$, where $v(\pi)=1, v(t)=0$, and the residue class of $t$ in $\bar{K}$ is a prime element of the latter field. The choice of such a system determines a rank two discrete valuation on $K$, and valuations obtained this way are equivalent. The group of principal units of $K$ with respect to any of these rank two valuations is denoted by $V_{K}$. Next, the group of principal units with respect to rank one valuation of $K$ is denoted by $U_{K}(1)$. In other words, $V_{K}$ (resp. $\left.U_{K}(1)\right)$ consists of all $a \in \mathcal{O}_{K}$ such that the residue class of $a$ in $\bar{K}$ belongs to $\mathcal{O}_{K}^{*}$ (resp. equals 1 ).

Proposition 0.3. Let $K$ be a two-dimensional local field (of characteristic either 0 or $p), k$ the constant subfield of $K$. Then $K$ is standard if and only if $K \simeq k\{\{t\}\}$.

Proof. Consider the embedding $k\{\{T\}\} \rightarrow K$, which maps $f(T)$ to $f(t)$. It is natural to denote its image by $k\{\{t\}\}$. Then $K / k\{\{t\}\}$ is a totally ramified extension with the ramification index $e_{K / k}$.

\subsection{Topological $K$-groups. (See [HLF, F3, Z2].)}

Well-known lemma 0.4.1. Let $F$ be a field, $\alpha, \beta \in F^{*}, \alpha, \beta \neq 0,1, \alpha \beta \neq \alpha-1$. Then

$$
\{1-\alpha, 1-\beta\}=\left\{\alpha(1-\beta), 1-\left(1-\alpha^{-1}\right)^{-1} \beta\right\}
$$

in $K_{2} F$.

Proof. Using the Steinberg relation, we obtain

$$
\begin{aligned}
\{1-\alpha, 1-\beta\} & =\{1-\alpha+\alpha \beta, 1-\beta\}-\left\{1+\frac{\alpha \beta}{1-\alpha}, 1-\beta\right\} \\
& =-\{1-\alpha+\alpha \beta, \alpha\}-\left\{1+\frac{\alpha \beta}{1-\alpha}, 1-\beta\right\} \\
& =-\left\{1+\frac{\alpha \beta}{1-\alpha}, \alpha\right\}-\left\{1+\frac{\alpha \beta}{1-\alpha}, 1-\beta\right\} \\
& =\left\{\alpha(1-\beta), 1+\frac{\alpha \beta}{1-\alpha}\right\} .
\end{aligned}
$$

We recall the definition and some properties of topological $K$-groups $K_{2}^{\text {top }} K$. 
Let $K$ be a two-dimensional local field. The topology on $K_{2} K$ is defined as the finest topology such that the natural map $K^{*} \times K^{*} \rightarrow K_{2} K$ is sequentially continuous, and subtraction in $K_{2} K$ is sequentially continuous. Then the intersection of all neighborhoods of zero in $K_{2} K$ coincides with $\cap_{l \geq 1} l K_{2} K$. The group $K_{2} K / \cap_{l \geq 1} l K_{2} K$ with the quotient topology is denoted by $K_{2}^{\text {top }} K$. When it does not lead to a confusion, the class of $\{a, b\} \in K_{2} K$ in $K_{2}^{\text {top }} K$ is also denoted by $\{a, b\}$.

Denote by $V K_{2}^{\text {top }} K$ (resp. $U(1) K_{2}^{\text {top }} K$ ) the subgroup of $K_{2}^{\text {top }} K$ generated by all symbols $\{u, x\}$, where $x \in K$ and $u \in V_{K}$ (resp. $u \in U_{K}(1)$ ).

Next, following [HLF, Ch. VI] and [F3], we give an alternative description of topology on $V K_{2}^{\text {top }} K$.

Let $\tau$ be a topology on a set $X$. The sequential saturation of $\tau$ is defined as the finest topology $\tau^{\prime}$ on $X$ such that the set of convergent sequences for $\tau^{\prime}$ is the same as that for $\tau$. For topological spaces $X, Y$ the $*$-product topology on $X \times Y$ is the sequential saturation of the product topology.

Theorem 0.4.2 ([HLF, 6.6; F3, Theorem 4.6]). Let $(t, \pi)$ be local parameters of $K$. Then the homomorphism

$$
\begin{aligned}
g: V_{K} \times V_{K} & \rightarrow V K_{2}^{\text {top }} K \\
(\alpha, \beta) & \mapsto\{\alpha, \pi\}+\{\beta, t\}
\end{aligned}
$$

induces a homeomorphism between $\left(V_{K} \times V_{K}\right) / \operatorname{Kerg}$ and $V K_{2}^{\text {top }} K$. Here $\left(V_{K} \times\right.$ $\left.V_{K}\right) / \operatorname{Kerg}$ is endowed with the quotient topology of *-product topology.

Corollary 0.4.3. Any neighborhood of 0 in $V K_{2}^{\text {top }} K$ contains $p^{n} V K_{2}^{\text {top }} K$ for some $n$.

Proof. Let $U$ be a neighborhood of 0 in $V K_{2}^{\text {top }} K$. If the claim is wrong, for any $i \geq 1$ there exists $a_{i} \in p^{i} V K_{2}^{\text {top }} K$ such that $a_{i} \notin U$. Let $a_{i}=p^{i} b_{i}, b_{i}=\left\{\alpha_{i}, \pi\right\}+\left\{\beta_{i}, t\right\}$, $\alpha_{i}, \beta_{i} \in V_{K}$. By Lemma 1.6 in [Z2], any neighborhood of 1 in $K$ contains $V_{K}^{p^{m}}$ for some $m$. Therefore, $\alpha_{i}^{p^{i}}, \beta_{i}^{p^{i}} \rightarrow 1$, whence $a_{i} \rightarrow 0$ in $V K_{2}^{\text {top }} K$ by Theorem 0.4.2.

Let $A$ be a subgroup in $V K_{2}^{\text {top }} K$. We denote by $\mathrm{Cl} A$ the intersection of all open subgroups containing $A$. This notation is justified by the fact that any closed subgroup in $V K_{2}^{\text {top }} K$ coincides with the intersection of all open subgroups containing it, as explained in [F3, Proof of Theorem 4.6]. Any set of generators of $A$ is said to be a set of topological generators for $\mathrm{Cl} A$. We say also that $A$ is dense in $\mathrm{Cl} A$.

Proposition 0.4.4. Let $L / K$ be a finite extension, $A$ any subgroup in $V K_{2}^{\text {top }} K$. Then $N_{L / K} \mathrm{Cl} A=\mathrm{Cl} N_{L / K} A$.

Proof. By [HLF, 6.8.2], the norm of any closed subgroup is closed, and, therefore, $N_{L / K} \mathrm{Cl} A \supset \mathrm{Cl} N_{L / K} A$.

Conversely, let $U^{\prime}$ be an open subgroup in $V K_{2}^{\text {top }} L$ containing $N_{L / K} A$. By Corollary 0.4.3, $U^{\prime}$ contains $p^{n} V K_{2}^{\text {top }} L$ for some $n$. Then $N_{L / K}^{-1}\left(U^{\prime}\right)$ is an open subgroup $V K_{2}^{\text {top }} K$; this is explained in [Z2, Corollary 4.4] and in [HLF, 6.8.2]. It follows $\mathrm{Cl} A \subset N_{L / K}^{-1}\left(U^{\prime}\right)$, and we conclude that $N_{L / K} \mathrm{Cl} A \subset \mathrm{Cl} N_{L / K} A$. 
Proposition 0.4.5. Let $K$ be a two-dimensional local field with the last residue field $F$, char $K=p$. Then any $a \in U(1) K_{2}^{\text {top }} K$ can be uniquely written in the form

$$
a=\sum_{\substack{i \geq 1, j \in \mathbb{Z} \\(j, p)=1 \\ \theta \in B}} c_{i j \theta}\left\{\pi, 1+\theta \pi^{i} t^{j}\right\}+\sum_{\substack{i \geq 1, j \in \mathbb{Z} \\(i, p)=1 \\(j, p) \neq 1 \\ \theta \in B}} c_{i j \theta}\left\{t, 1+\theta \pi^{i} t^{j}\right\}
$$

where $B$ is a certain fixed $\mathbb{F}_{p}$-basis of $F, c_{i j \theta} \in \mathbb{Z}_{p}$. Here, for any positive integer $n$, the set $\left\{(j, i) \mid v_{p}\left(c_{i j \theta}\right)<n\right.$ for some $\left.\theta\right\}$ is admissible, $i . e ., p^{n} \mid c_{i j \theta}$ for all sufficiently small $j<j(i)$ and any $\theta$.

For the existence and uniqueness of expansion, see [P, $\S 2$, Prop. 1 and 3$]$ when $F$ is finite, and [F2, Prop. 2.4] in the general case. The remaining statement follows from [MZ2]; we shall not use it.

\section{$\S 1$. General Ramification theory}

\subsection{Definitions.}

For the whole paper, we denote

$$
\mathbb{I}=\{-1,0\} \cup\{(\mathbf{c}, i) \mid i \in \mathbb{Q}, i>0\} \cup\{(\mathbf{c}, \infty)\} \cup\{(\mathbf{i}, i) \mid i \in \mathbb{Q}, i>0\} \cup\{\infty\}
$$

This is the index set for lower and upper numbering of ramification subgroups we are going to introduce. (The letters $\mathbf{c}$ and $\mathbf{i}$ are related to (almost) constant and infernal extensions respectively.) This set is linearly ordered as follows:

$$
\begin{gathered}
-1<0<(\mathbf{c}, i)<(\mathbf{i}, j)<\infty \text { for any } i, j \\
(\mathbf{c}, i)<(\mathbf{c}, j) \text { for any } i<j \\
(\mathbf{i}, i)<(\mathbf{i}, j) \text { for any } i<j .
\end{gathered}
$$

Let $K$ be a complete discrete valuation field of any characteristic with the residue field $\bar{K}$ of characteristic $p>0$. We assume that $\left[\bar{K}: \bar{K}^{p}\right]=p$.

Let $L / K$ be a finite Galois extension, $G=\operatorname{Gal}(L / K)$. For any $\alpha \in \mathbb{I}$ we are going to define a subgroup $G_{\alpha}$ in $G$.

We put $G_{-1}=G$, and denote by $G_{0}$ the inertia subgroup in $G$, i. e.,

$$
G_{0}=\left\{g \in G \mid g(a)-a \in \mathfrak{M}_{L} \text { for all } a \in \mathcal{O}_{L}\right\}
$$

Denote by $K_{\mathbf{c}} / K$ the maximal almost constant subextension in $L / K$. To introduce subgroups $G_{(\mathbf{c}, i)}=G_{\mathbf{c}, i}$, we consider first the case when $K_{\mathbf{c}} / K$ is constant and contains no unramified subextension. Then $K_{\mathbf{c}}=l K$, and we have a natural projection

$$
\text { pr: } \operatorname{Gal}(L / K) \rightarrow \operatorname{Gal}\left(K_{\mathbf{c}} / K\right)=\operatorname{Gal}(l / k)=\operatorname{Gal}(l / k)_{0},
$$

where $l$ and $k$ are the constant subfields in $L$ and $K$ respectively. Then we put $G_{\mathbf{c}, i}=\operatorname{pr}^{-1}\left(\operatorname{Gal}(l / k)_{i}\right)$. In the general case take an unramified extension $K^{\prime} / K$ such that $K^{\prime} L / K^{\prime}$ contains no unramified subextension, and the maximal almost 
constant subextension in $K^{\prime} L / K^{\prime}$ (i. e., $K^{\prime} K_{\mathbf{c}} / K^{\prime}$ ) is constant. We put $G_{\mathbf{c}, i}=$ $\operatorname{Gal}\left(K^{\prime} L / K^{\prime}\right)_{\mathbf{c}, i}$. It is easy to see that the choice of $K^{\prime}$ plays no role.

Next,

$$
G_{\mathbf{c}, \infty}=\operatorname{Gal}\left(L / K_{\mathbf{c}}\right)=G_{\mathbf{c}, m} .
$$

for $m$ big enough. Now we turn to the definition of $G_{(\mathbf{i}, i)}=G_{\mathbf{i}, i}, i>0$.

Assume that $K_{\mathbf{c}}$ is standard and $L / K_{\mathbf{c}}$ is ferociously ramified. Let $t \in \mathcal{O}_{L}$, $\bar{t} \notin \bar{L}^{p}$. We define

$$
G_{\mathbf{i}, i}=\left\{g \in \operatorname{Gal}\left(L / K_{\mathbf{c}}\right) \mid v_{0}(g(t)-t) \geq i\right\}
$$

for all $i>0$.

We recall that $v_{0}=v_{k_{0}}$; thus, $v_{0}\left(L^{*}\right)=e_{L}^{-1} \mathbb{Z}$. Notice that in the case under consideration $G_{\mathbf{i}, i}=G_{i e_{L}-1}$ in the notation of [FV]. This follows directly from the definition of usual ramification groups in [FV, (4.3)]. Therefore, $G_{\mathbf{i}, i}$ is independent from the choice of $t$.

In the general case choose a finite extension $l^{\prime} / l$ such that $l^{\prime} K_{\mathbf{c}}$ is standard and $e\left(l^{\prime} L / l^{\prime} K_{\mathbf{c}}\right)=1$, see Proposition 0.2.2 and Corollary. Then it is clear that $\operatorname{Gal}\left(l^{\prime} L / l^{\prime} K_{\mathbf{c}}\right)=\operatorname{Gal}\left(L / K_{\mathbf{c}}\right)$. Next, $l^{\prime} L / l^{\prime} K_{\mathbf{c}}$ is infernal, whence there is no nontrivial unramified subextension in $l^{\prime} L / l^{\prime} K_{\mathbf{c}}$. Together with $e\left(l^{\prime} L / l^{\prime} K_{\mathbf{c}}\right)=1$ this means that $l^{\prime} L / l^{\prime} K_{\mathbf{c}}$ is ferociously ramified. We define

$$
G_{\mathbf{i}, i}=\operatorname{Gal}\left(l^{\prime} L / l^{\prime} K_{\mathbf{c}}\right)_{\mathbf{i}, i}=\operatorname{Gal}\left(l^{\prime} L / l^{\prime} K\right)_{\mathbf{i}, i}
$$

for all $i>0$.

Finally, put $G_{\infty}=\{e\}$.

Proposition 1.1.1. $G_{\mathbf{i}, i}$ does not depend on the choice of $l^{\prime}$.

Proof. Let $l^{\prime} / l$ and $l^{\prime \prime} / l$ be finite extensions such that $l^{\prime} K_{\mathbf{c}}$ and $l^{\prime \prime} K_{\mathbf{c}}$ are standard, and

$$
e\left(l^{\prime} L / l^{\prime} K_{\mathbf{c}}\right)=e\left(l^{\prime \prime} L / l^{\prime \prime} K_{\mathbf{c}}\right)=1 .
$$

Then $l^{\prime} l^{\prime \prime} K_{\mathbf{c}}$ is standard, and $e\left(l^{\prime} l^{\prime \prime} L / l^{\prime} l^{\prime \prime} K_{\mathbf{c}}\right)=1$. We may therefore assume that $l^{\prime} \subset l^{\prime \prime}$. Let $t \in \mathcal{O}_{l^{\prime} L}, \bar{t} \notin\left(\overline{l^{\prime} L}\right)^{p}$. Then we have $t \in \mathcal{O}_{l^{\prime \prime} L}, \bar{t} \notin\left(\overline{l^{\prime \prime} L}\right)^{p}$, and we conclude that $\operatorname{Gal}\left(l^{\prime \prime} L / l^{\prime \prime} K_{\mathbf{c}}\right)_{\mathbf{i}, i}=\operatorname{Gal}\left(l^{\prime} L / l^{\prime} K_{\mathbf{c}}\right)_{\mathbf{i}, i}$ immediately by the definition of $G_{\mathbf{i}, i}$ in the ferociously ramified case.

Thus, we have defined $G_{\alpha}$ for all $\alpha \in \mathbb{I}$, and we see immediately that $\alpha<\beta$ implies $G_{\alpha} \supseteq G_{\beta}$. We say that $\alpha \in \mathbb{I}$ is a jump for $L / K$ if $G_{\alpha} \neq G_{\beta}$ for any $\beta>\alpha$.

Proposition 1.1.2. For any $\alpha \in \mathbb{I}, G_{\alpha}$ is a normal subgroup in $G$.

Proof. We have nothing to prove, if $\alpha=-1$ or $\alpha=\infty$. It is well known that $G_{0}$ is normal, see, e. g., [FV, (4.3)]. Next, let $\alpha=(\mathbf{c}, i)$. We may assume that $K^{\prime} / K$ in the definition of $G_{\mathbf{c}, i}$ is normal. Let $g \in G, h \in G_{\alpha}$. Extend $g$ and $h$ onto $K^{\prime} L / K$ so that $h \in \operatorname{Gal}\left(K^{\prime} L / K^{\prime}\right)$, this can be done since $h \in G_{\alpha} \subset G_{0}$. We see

$$
\left.g h g^{-1}\right|_{K^{\prime}}=\left.\left.g\right|_{K^{\prime}} \cdot g^{-1}\right|_{K^{\prime}}=e,
$$

whence $g h g^{-1} \in \operatorname{Gal}\left(K^{\prime} L / K^{\prime}\right)=G_{0}$. 
Let $K_{\mathbf{c}}^{\prime} / K$ be the maximal almost constant subextension in $K^{\prime} L / K^{\prime}$, and let $k^{\prime}$, $l^{\prime}$ be the constant subfields in $K^{\prime}$ and $K^{\prime} L$ respectively. We have $g\left(k^{\prime}\right)=k^{\prime}$, whence $g\left(K_{\mathbf{c}}^{\prime}\right)=K_{\mathbf{c}}^{\prime}$. Now

$\operatorname{pr}\left(g h g^{-1}\right)=\left.\left.\left.g\right|_{K_{\mathbf{c}}^{\prime}} \cdot h\right|_{K_{\mathbf{c}}^{\prime}} \cdot g^{-1}\right|_{K_{\mathbf{c}}^{\prime}} \in g \operatorname{Gal}\left(l^{\prime} / k^{\prime}\right)_{i} g^{-1}=\operatorname{Gal}\left(g\left(l^{\prime}\right) / g\left(k^{\prime}\right)\right)_{i}=\operatorname{Gal}\left(l^{\prime} / k^{\prime}\right)_{i}$.

This proves also the case $\alpha=(\mathbf{c}, \infty)$.

In the case $\alpha=(\mathbf{i}, i)$ the same argument is applicable. Indeed, $l^{\prime} / l$ in the definition may be chosen to be normal, and the relation $\left.v_{0}(g(t)-t)\right) \geq i$ is stable with respect to any automorphisms of discretely valued fields.

\subsection{Compatibility with subgroups.}

Proposition 1.2. Let $H$ be a subgroup in $G$. Then for any $\alpha \in \mathbb{I}$ we have $H_{\alpha}=$ $H \cap G_{\alpha}$.

Proof. The assertion is obvious for $\alpha=-1,0, \infty$. Next, denote by $M$ the intermediate field in $L / K$ which corresponds to $H$. Then $K_{\mathbf{c}} M / M$ is almost constant, whence $K_{\mathbf{c}} M \subset M_{\mathbf{c}}$. On the other hand, $L / K_{\mathbf{c}} M$ is infernal, and we conclude $K_{\mathbf{c}} M=M_{\mathbf{c}}$. This proves the assertion for $\alpha=(\mathbf{c}, \infty)$. Next, if $e\left(\tilde{l} L / \tilde{l} K_{\mathbf{c}}\right)=1$, then $e\left(\tilde{l} L / \tilde{l} M_{\mathbf{c}}\right)=1$ as well. It is clear now that

$$
H_{\mathbf{i}, i}=\operatorname{Gal}\left(L / M_{\mathbf{c}}\right) \cap G_{\mathbf{i}, i}=H_{\mathbf{c}, \infty} \cap G_{\mathbf{i}, i}=H \cap G_{\mathbf{i}, i}
$$

for all $i>0$.

Finally, let $\alpha=(\mathbf{c}, i)$. Denote by $K^{\prime} / K$ any unramified extension such that $K^{\prime} L / K^{\prime}$ contains no unramified subextension, and the maximal almost constant subextension in $K^{\prime} L / K^{\prime}$ is constant. Then $K^{\prime} L / K^{\prime} M$ possesses these two properties as well. Let $k^{\prime}, l^{\prime}, m^{\prime}$ be constant subfields in $K^{\prime}, K^{\prime} L, K^{\prime} M$ respectively. Then the natural projection $\operatorname{Gal}\left(K^{\prime} L / K^{\prime} M\right) \rightarrow \operatorname{Gal}\left(l^{\prime} / m^{\prime}\right)$ is the restriction of the natural projection pr: $\operatorname{Gal}\left(K^{\prime} L / K^{\prime}\right) \rightarrow \operatorname{Gal}\left(l^{\prime} / k^{\prime}\right)$. Therefore,

$$
\begin{aligned}
H_{\alpha} & =H \cap \operatorname{pr}^{-1}\left(\operatorname{Gal}\left(l^{\prime} / m^{\prime}\right)_{i}\right) \\
& =H \cap \operatorname{pr}^{-1}\left(\operatorname{Gal}\left(l^{\prime} / m^{\prime}\right) \cap \operatorname{Gal}\left(l^{\prime} / k^{\prime}\right)_{i}\right) \\
& =H \cap \operatorname{Gal}\left(K^{\prime} L / m^{\prime} K^{\prime}\right) \cap \operatorname{Gal}\left(K^{\prime} L / K^{\prime}\right)_{\alpha} \\
& =H \cap G_{\alpha},
\end{aligned}
$$

the latter equality follows from

$$
H \cap \operatorname{Gal}\left(K^{\prime} L / K^{\prime}\right)_{\alpha} \subset H \cap \operatorname{Gal}\left(K^{\prime} L / K^{\prime}\right)=\operatorname{Gal}\left(K^{\prime} L / K^{\prime} M\right) \subset \operatorname{Gal}\left(K^{\prime} L / m^{\prime} K^{\prime}\right) .
$$

\subsection{Hasse-Herbrand functions and compatibility with quotient groups.}

To compute the ramification of a quotient group $G / H$, we have to express the ramification number of given $\sigma \in G / H$ in terms of ramification numbers of its representatives in $G$. For constant extensions, we use [Se, Ch. IV, Prop. 3 and Lemma 5]. Now we carry out the same calculation for the case of ferociously ramified $L / K$.

Let $t \in \mathcal{O}_{L}, \bar{t} \notin \bar{L}^{p}$. Let

$$
i_{G}(g)=v_{0}(g(t)-t)=\max \left\{i \mid g \in G_{\mathbf{i}, i}\right\}
$$

for $g \in G=\operatorname{Gal}(L / K), g \neq 1$. 
Lemma 1.3.1. Let $L / K$ be ferociously ramified, $H$ a normal subgroup in $G, \sigma \in$ $G / H, \sigma \neq 1$. Then

$$
i_{G / H}(\sigma)=i_{G}\left(g_{1}\right)+\cdots+i_{G}\left(g_{n}\right),
$$

where $g_{1}, \ldots, g_{n}$ are all the representatives of $\sigma$ in $G$.

Proof. Similarly to [Se, Ch. IV, Prop. 3].

Let $L / K$ be an arbitrary finite Galois extension, $K_{\mathbf{c}} / K$ the maximal almost constant subextension in $L / K$. We define $\Phi_{L / K}: \mathbb{I} \rightarrow \mathbb{I}$ as follows:

$$
\begin{aligned}
\Phi_{L / K}(\alpha) & =\alpha \text { for } \alpha=-1,0,(\mathbf{c}, \infty), \infty \\
\Phi_{L / K}((\mathbf{c}, i)) & =\left(\mathbf{c}, \frac{1}{e(L / K)} \int_{0}^{i}\left|\operatorname{Gal}\left(K_{\mathbf{c}} / K\right)_{\mathbf{c}, t}\right| d t\right) \text { for all } i>0 ; \\
\Phi_{L / K}((\mathbf{i}, i)) & =\left(\mathbf{i}, \int_{0}^{i}\left|\operatorname{Gal}(L / K)_{\mathbf{i}, t}\right| d t\right) \text { for all } i>0 .
\end{aligned}
$$

It is easily seen that $\Phi_{L / K}$ is bijective and increasing; we introduce also $\Psi_{L / K}=$ $\Phi_{L / K}^{-1}$.

Proposition 1.3.2. Let $L / K$ and $M / K$ be finite Galois extensions with $M \subset L$. Then $\Phi_{L / K}=\Phi_{M / K} \circ \Phi_{L / M}$.

Proof. The proposition follows immediately from the definitions in the following three cases: $L / K$ is almost constant; $L / K$ is infernal (by Lemma 1.3.1); $M / K$ is almost constant whereas $L / M$ is infernal.

Let now $M / K$ be infernal, and $L / M$ be almost constant. Then there exists an almost constant Galois extension $L^{\prime} / K$ such that $L=L^{\prime} M$. (The argument is the same as in the proof of the 1st property of infernal extension in 0.2.) The extension $L^{\prime} / K$ is in fact the maximal almost constant subextension in $L / K$. By the definition $\Phi_{M / K}=\Phi_{L / L^{\prime}}$. It is also easy to see that $\Phi_{L^{\prime} / K}=\Phi_{L / M}$. These two functions commute since $\Phi_{M / K}$ affects only the "infernal" part of $\mathbb{I}$, and $\Phi_{L^{\prime} / K}$ only the "constant" one. Therefore,

$$
\Phi_{M / K} \circ \Phi_{L / M}=\Phi_{L / L^{\prime}} \circ \Phi_{L^{\prime} / K}=\Phi_{L^{\prime} / K} \circ \Phi_{L / L^{\prime}}=\Phi_{L / K}
$$

In the general case let $T / M$ be the maximal almost constant subextension in $L / M$, and $S / K$ that in $M / K$. This is clear that $T / S$ is a Galois extension. Let $T^{\prime} / S$ be the maximal almost constant subextension in it. Then $T^{\prime}$ is obviously the maximal almost constant subextension also in $L / K$ and, therefore, normal over $K$. We have now

$$
\begin{aligned}
\Phi_{L / K} & =\Phi_{L / T^{\prime}} \circ \Phi_{T^{\prime} / K} \\
& =\Phi_{L / T} \circ \Phi_{T / T^{\prime}} \circ \Phi_{T^{\prime} / S} \circ \Phi_{S / K} \\
& =\Phi_{L / T} \circ \Phi_{T / S} \circ \Phi_{S / K} \\
& =\Phi_{L / T} \circ \Phi_{T / M} \circ \Phi_{M / S} \circ \Phi_{S / K} \\
& =\Phi_{L / M} \circ \Phi_{M / K} \cdot \quad \square
\end{aligned}
$$


Proposition 1.3.3. Let $G=\operatorname{Gal}(L / K), H$ a normal subgroup in $G, M$ the corresponding intermediate field. Then for any $\alpha \in \mathbb{I}$ we have $(G / H)_{\alpha}=G_{\Psi_{L / M}(\alpha)} H / H$.

Proof. The cases $\alpha=-1, \alpha=\infty$ are trivial.

Let $\alpha=0$. If $g \in G_{0}$, then $g$ acts trivially on $\bar{M} \subset \bar{L}$, whence $g H \in(G / H)_{0}$. Conversely, let $g H \in(G / H)_{0}$. Then the image of $g$ in $\operatorname{Gal}(\bar{L} / \bar{K})$ is $\bar{h} \in \operatorname{Gal}(\bar{L} / \bar{M})$. If $h$ is a preimage of $\bar{h}$ in $H=\operatorname{Gal}(L / M)$, then $g h^{-1} \in G_{0}$, whence $g H \in G_{0} H / H$.

Let $\alpha=(\mathbf{c}, i), 0<i \leq \infty$. Consider first the case when $K_{\mathbf{c}} / K$ is constant and contains no unramified subextensions. Let $k, l, m$ be the constant subfields of $K, L, M$ respectively. Consider the commutative diagram of natural epimorphisms

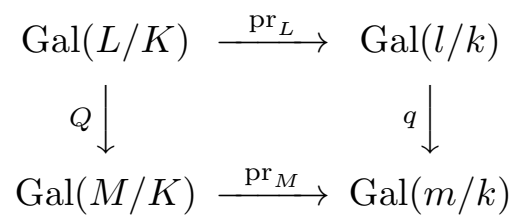

It is sufficient to prove that $Q^{-1}\left((G / H)_{\alpha}\right)=G_{\Psi_{L / M}(\alpha)} H$. We have

$$
\begin{aligned}
Q^{-1}\left((G / H)_{\alpha}\right) & =Q^{-1}\left(\operatorname{pr}_{M}^{-1}\left(\operatorname{Gal}(m / k)_{i}\right)\right) \\
& =\operatorname{pr}_{L}^{-1}\left(q^{-1}\left(\operatorname{Gal}(m / k)_{i}\right)\right) \\
& =\operatorname{pr}_{L}^{-1}\left(q^{-1}\left(\operatorname{Gal}(l / k)_{\psi_{l / m}(i)} \operatorname{Gal}(l / m) / \operatorname{Gal}(l / m)\right)\right) \\
& =\operatorname{pr}_{L}^{-1}\left(\operatorname{Gal}(l / k)_{\psi_{l / m}(i)} \operatorname{Gal}(l / m)\right) \\
& =G_{\mathbf{c}, \psi_{l / m}(i)} \cdot \operatorname{Gal}(L / m K) \\
& =G_{\Psi_{L / M}(\alpha)} \cdot \operatorname{Gal}(L / m K) \\
& \supset G_{\Psi_{L / M}(\alpha)} H
\end{aligned}
$$

It remains to prove that $\operatorname{Gal}(L / m K) \subset G_{\Psi_{L / M}(\alpha)} H$. Let $g \in \operatorname{Gal}(L / m K)$, then $\operatorname{pr}_{L}(g) \in \operatorname{Gal}(l / m)$. Let $h$ be any preimage of $\operatorname{pr}_{L}(g)$ in $H=\operatorname{Gal}(L / M)$; then $g h^{-1} \in \operatorname{Gal}(L / l K)=G_{\mathbf{c}, \infty} \subset G_{\psi_{L / M}(\alpha)}$.

In the general case take $K^{\prime} / K$ as in the proof of Prop. $1.2 ; H^{\prime}=\operatorname{Gal}\left(K^{\prime} L / K^{\prime} M\right)$. Then $\Psi_{L / M}=\Psi_{K^{\prime} L / K^{\prime} M}$, and

$$
\operatorname{Gal}\left(K^{\prime} M / K^{\prime}\right)_{\alpha}=\operatorname{Gal}\left(K^{\prime} L / K^{\prime}\right)_{\Psi_{L / M}(\alpha)} H^{\prime} / H^{\prime} .
$$

The natural embedding $\operatorname{Gal}\left(K^{\prime} M / K^{\prime}\right) \hookrightarrow G / H$ identifies these groups with $(G / H)_{\alpha}$ and $G_{\Psi_{L / M}(\alpha)} H / H$ respectively.

Finally, let $\alpha=(\mathbf{i}, i)$. Denote by $K_{\mathbf{c}}^{L}$ and $K_{\mathbf{c}}^{M}$ the maximal almost constant extensions in $L / K$ and $M / K$ respectively; we have $K_{\mathbf{c}}^{M} \subset K_{\mathbf{c}}^{L}$. Consider the case when both $K_{\mathbf{c}}^{L}$ and $K_{\mathbf{c}}^{M}$ are standard, and $L / K_{\mathbf{c}}^{M}$ is ferociously ramified. In this case we use Lemma 1.3.1 and argue exactly as in [Se, Ch. IV, Lemmas $3,4,5]$. In the general case take a finite extension $l^{\prime} / l$ such that $l^{\prime} K_{\mathbf{c}}^{M}$ and $l^{\prime} K_{\mathbf{c}}^{L}$ are standard, and $e\left(l^{\prime} L / l^{\prime} K_{\mathbf{c}}^{M}\right)=1$. Then $G_{\Psi_{L / M}(\alpha)}=\operatorname{Gal}\left(l^{\prime} L / l^{\prime} K\right)_{\Psi_{L / M}(\alpha)}$, and $(G / H)_{\alpha}=\operatorname{Gal}\left(l^{\prime} M / l^{\prime} K\right)_{\alpha}$. Since $\Psi_{L / M}(\alpha)=\Psi_{l^{\prime} L / l^{\prime} M}(\alpha)$ for $\alpha \geq(\mathbf{c}, \infty)$, we have $\operatorname{Gal}\left(l^{\prime} M / l^{\prime} K\right)_{\alpha}=\operatorname{Gal}\left(l^{\prime} L / l^{\prime} K\right)_{\Psi_{L / M}(\alpha)} H^{\prime} / H^{\prime}$, where $H^{\prime}=\operatorname{Gal}\left(l^{\prime} L / l^{\prime} M\right)$; in $G / H$ this yields $(G / H)_{\alpha}=G_{\psi_{L / M}(\alpha)} H / H$ 


\subsection{Upper numbering.}

As usual, we denote $G^{\alpha}=G_{\Psi_{L / K}(\alpha)}$ for any $\alpha \in \mathbb{I}$. Then Proposition 1.3.3 can be reformulated as follows.

Corollary 1.4. Let $G$ be the Galois group of a finite Galois extension of $K, H a$ normal subgroup in $G$. Then for any $\alpha \in \mathbb{I}$ we have $(G / H)^{\alpha}=G^{\alpha} H / H$.

Let $L / K$ be an arbitrary Galois extension, $L=\bigcup L_{i}, L_{i} / K$ finite Galois extensions, $G=\operatorname{Gal}(L / K)$. Then we define

$$
G^{\alpha}=\lim _{\leftarrow} \operatorname{Gal}\left(L_{i} / K\right)^{\alpha} ; \quad L^{\alpha}=L^{G^{\alpha}}
$$

Further, one can define $K_{\alpha}=\left(K^{\text {sep }}\right)^{\alpha}$ for all $\alpha \in \mathbb{I}$. Then $K_{-1}=K, K_{0}=K^{u r}$, $K_{\mathbf{c}, 1}=K^{t r}, K_{\mathbf{c}, \infty}=K^{u r} k^{\mathrm{sep}}, K_{\infty}=K^{\mathrm{sep}}$, where $k$ is the constant subfield in $K$.

\subsection{Examples.}

In all examples $K=F((\pi))$ is of characteristic $p$, standard, with $\pi \in k ; t$ is an element of $F \backslash F^{p}$. Assume $v_{0}(\pi)=1$.

1. Let $L$ be the splitting field of $X^{p}-X-\pi^{-p i} t$, where $i>0$. Then the only jump of lower filtration for $L / K$ is $(\mathbf{i}, i)$ and the only jump of upper filtration is $(\mathbf{i}, p i)$.

Indeed, $L / K$ is ferociously ramified, and $\mathcal{O}_{L}=\mathcal{O}_{K}\left[\pi^{i} x\right]$, where $x$ is a root of $X^{p}-X-\pi^{-p i} t$.

2. Let $L$ be the splitting field of $X^{p}-X-\pi^{-i} t$ where $i>0$, and $i$ is prime to $p$. Then the only jump of lower filtration is $(\mathbf{i}, i / p)$ and the only jump of upper filtration is $(\mathbf{i}, i)$.

In this case $L / K$ is infernal but totally ramified. To compute the ramification, consider $l^{\prime}=k\left(\pi_{1}\right), \pi_{1}^{p}=\pi$. Then $l^{\prime} L / l^{\prime} K$ is ferociously ramified, and the minimal polynomial of this extension is $X^{p}-X-\pi_{1}^{-p i} t$. Now we apply the previous example and take into account that $v_{0}\left(\pi_{1}\right)=p^{-1}$.

3. Let $L$ be the splitting field of $X^{p}-X-\pi^{-i} t^{p^{j}}$ where $i>0$, and $i$ is prime to $p$. Then the only jump of lower filtration is $\left(\mathbf{i}, i / p^{j+1}\right)$ and the only jump of upper filtration is $\left(\mathbf{i}, i / p^{j}\right)$.

In this case take $l^{\prime}=k\left(\pi_{j+1}\right), \pi_{j+1}^{p^{j+1}}=\pi$. Then $\pi^{-i} t^{p^{j}} \equiv \pi_{j+1}^{-p i} t \bmod \wp\left(l^{\prime} K\right)$. Therefore, $l^{\prime} L=l^{\prime} K(x)$, where $x$ is a root of $X^{p}-X-\pi_{j+1}^{-p i} t$. We conclude that $l^{\prime} L / l^{\prime} K$ is ferociously ramified, and the jumps can be computed as in the first example.

4. Let $L$ be the splitting field of $X^{p}-X-\pi^{-i}$ where $i>0$, and $i$ is prime to $p$. Then the only jump of lower filtration is $(\mathbf{c}, i)$ and the only jump of upper filtration is $(\mathbf{c}, i)$.

\section{$\S 2$. EXAmple: ABelian eXtensions of EXPONEnt $p$}

We start with the case char $K=p$. In this case the group of characters of $\operatorname{Gal}(K)$ of exponent $p$ can be identified via Artin-Schreier theory with the group $K / \wp(K)$, where $\operatorname{Gal}(K)$ is the absolute Galois group of $K$, and $\wp: K \rightarrow K$ is the Artin-Schreier map $x \mapsto x^{p}-x$. Since the group $\operatorname{Gal}(K)$ possesses a decreasing filtration indexed by $\mathbb{I}$, the additive group of $K$ acquires an increasing filtration indexed by $\mathbb{I}$. 
Let $\alpha \in \mathbb{I}$. Denote by $A_{\alpha}=A_{\alpha, K}$ the set of such $a \in K$ that the polynomial $X^{p}-X-a$ completely splits in $K_{\alpha}$. These $A_{\alpha}$ form an increasing filtration on $K$ :

$$
\wp(K)=A_{-1} \subseteq A_{0} \subseteq A_{\mathbf{c}, 1} \subseteq \cdots \subseteq A_{\infty}=K .
$$

It is obvious that $A_{0}=\mathcal{O}_{K}+\wp(K)$. Further, it is easy to obtain that $A_{\mathbf{c}, i}=$ $\mathfrak{M}_{k}^{-i}+A_{0}$ for all integral $i>0$, and $A_{\mathbf{c}, \infty}=k+A_{0}$. As for $A_{\mathbf{i}, i}$, these subgroups can be calculated explicitly only in case $K$ is standard.

Proposition. Let $K$ be standard, $i$. e., $K=F((\pi))$ with $\pi \in k$. Then

$$
A_{\mathbf{i}, i}=k+A_{0}+\sum_{l=0}^{\infty} \pi^{-p^{l} i} F^{p^{l}}[[\pi]] .
$$

Proof. Let $a$ belong to the right hand side of (1), $x$ be a root of $X^{p}-X-a$. To prove that $K(x) \subset K_{\mathbf{i}, i}$, it is sufficient to consider the following cases.

1. $a \in k$, then $K(x) / K$ is constant, whence $K(x) \subset K_{\mathbf{c}, \infty} \subset K_{\mathbf{i}, i}$.

2. $a \in \mathcal{O}_{K}$, then $K(x) / K$ is unramified, whence $K(x) \subset K_{0} \subset K_{\mathbf{i}, i}$.

3. $a \in \pi^{-p^{l} i} F^{p^{l}}[[\pi]]$. In the field $K^{\prime}=K\left(\pi^{\prime}\right)$, where $\pi^{p^{l}}=\pi$, we have $a=b^{p^{l}}$, $b \in \pi^{\prime-p^{l} i} F\left[\left[\pi^{\prime}\right]\right]$. Observe that $b^{p^{l}} \equiv b \bmod \wp\left(K^{\prime}\right)$, whence $K^{\prime}(x)=K^{\prime}\left(x^{\prime}\right)$, $x^{\prime p}-x^{\prime}=b$. Clearly, $v_{0}\left(x^{\prime}\right)=p^{-1} v_{0}(b) \geq-p^{-1} i$. Without loss of generality we may assume that $K(x) / K$ is infernal. By Proposition 0.2.1, there exists a finite constant extension $K^{\prime \prime} / K^{\prime}$ such that $K^{\prime \prime}(x) / K^{\prime \prime}$ is ferociously ramified. If $g$ is a generator of $\operatorname{Gal}\left(K^{\prime \prime}(x) / K^{\prime \prime}\right)$, we have

$$
v_{0}\left(g\left(x^{\prime}\right)-x^{\prime}\right)=0
$$

whence the only jump in the lower filtration is $\leq\left(\mathbf{i}, p^{-1} i\right)$, and the only jump in the upper filtration is $\leq(\mathbf{i}, i)$. We conclude $K(x) \subset K_{\mathbf{i}, i}$, and $a \in A_{\mathbf{i}, i}$.

Conversely, suppose that $a \in A_{\mathbf{i}, i}, x^{p}-x=a$. If $K(x) / K$ is almost constant, then $a=a_{u}+a_{c}$, such that $K\left(x_{u}\right) / K$ is unramified and $K\left(x_{c}\right) / K$ is constant, $x_{c}^{p}-x_{c}=a_{c} \in K, x_{u}^{p}-x_{u}=a_{u} \in K$. It follows $a_{c} \in k+\wp(K), a_{u} \in \mathcal{O}_{K}+\wp(K)$, and we are done.

It remains to consider the case of infernal $K(x) / K$. Let

$$
a=b_{m} \pi^{-m}+b_{m-1} \pi^{-(m-1)}+\ldots,
$$

where $b_{j} \in F$. Adding an element of $k \subset A_{\mathbf{c}, \infty} \subset A_{\mathbf{i}, i}$ to $a$, we may assume $b_{j}=d_{j}^{p_{j}}$, where $d_{j} \notin F^{p}$. Similarly, adding an element of $\wp(K)$, we may assume that for any $j$ either $p \nmid j$ or $l_{j}=0$. Consider quotients $\varkappa_{j}=e_{K}^{-1} j / p^{l_{j}}$. ( $e_{K}$ plays the role of absolute ramification index.) Due to the previous remark, the values of $\varkappa_{j}$ are all different. $\left(\varkappa_{r}=\varkappa_{s}\right.$ would imply that at least one of the fractions $r / p^{l_{r}}$ and $s / p^{l_{s}}$ is reducible.) Notice that $\varkappa=\max \left(\varkappa_{j}\right)$ remains invariant when we replace $K$ with $K(\sqrt[p]{\pi})$.

Now let $K^{\prime}=K\left(\pi^{\prime}\right)$, where $\pi^{p^{N}}=\pi, N=\max \left(l_{j}\right)+1$. Then it is easy to see that $K^{\prime}(x)=K^{\prime}\left(x^{\prime}\right)$,

$$
x^{\prime p}-x^{\prime}=\pi^{\prime-\varkappa e_{K^{\prime}}} d+\ldots,
$$


$d \notin F^{p}$. Since $p \mid \varkappa e_{K^{\prime}}$, we conclude that $K^{\prime}(x) / K$ is ferociously ramified, and the jump of ramification in upper numbering is $\varkappa$. This means $\varkappa \leq i$, and $a$ lies in the right hand side of (1).

Now we turn to the case where char $K=0$ and $K$ contains all $p$ th roots of unity.

Let $\alpha \in \mathbb{I}$. Denote by $B_{\alpha}$ the set of such $b \in K^{*}$ that $\sqrt[p]{b} \in K_{\alpha}$. These $B_{\alpha}$ form an increasing filtration in $K^{*}$ :

$$
\left(K^{*}\right)^{p}=B_{-1} \subseteq B_{0} \subseteq B_{\mathbf{c}, 1} \subseteq \cdots \subseteq B_{\infty}=K^{*} .
$$

It is obvious that $B_{0}=U_{\frac{p e}{p-1}, K} \cdot\left(K^{*}\right)^{p}$, where $e=e_{K}$. Further, it is easy to obtain that

$$
B_{\mathbf{c}, i}= \begin{cases}U_{\frac{p e}{p-1}-i, k} B_{0}, & i<\frac{p e}{p-1}, \\ k^{*} B_{0}, & i \geq \frac{p e}{p-1} .\end{cases}
$$

If $K$ is standard then $B_{\mathbf{i}, i}$ is generated by $k^{*} B_{0}$ and all elements of the kind $1+\pi^{j} a^{p^{m}}$ where $j \geq 0, a \in \mathcal{O}_{K}$ and $m$ is the minimal non-negative integer such that

$$
\frac{p e}{p-1}-j \leq p^{m} i
$$

\section{§3. Fine RAMificATION THEORY}

Assume that the algebraic closure of the residue field of $K$ is also endowed with a valuation $w:\left(\bar{K}^{\mathrm{sep}}\right)^{*} \rightarrow \mathbb{Q}$ such that the restriction of $w$ to $\bar{K}$ is discrete. It is clear that the restriction of $w$ to the maximal perfect subfield of $\bar{K}$ is trivial. The main example is given by a two-dimensional local field $K$. In this case $\bar{K}$ is the field of Laurent power series over a perfect field $F$. One can take for $w$ the extension of the valuation on $\bar{K}$ onto $\bar{K}^{\text {sep }}$. Another example is a two-dimensional local-global field, i. e., $\bar{K}$ is a field of algebraic functions in one variable over a perfect field. (Then there is a lot of non-equivalent valuations $w$.)

Now we define the canonical (up to the choice of a base subfield and of $w$ ) rank 2 valuation $\mathbf{v}_{0}$ on $K$.

Proposition 3.1. Let $k$ be the constant subfield in $K$. There exists a unique valuation $\mathbf{v}_{0}=\left(v_{1}, v_{2}\right):\left(K^{\mathrm{alg}}\right)^{*} \rightarrow \mathbb{Q} \times \mathbb{Q}$ such that $v_{2}(a)=v_{0}(a)$ for all $a \in K^{*}$, $v_{1}(u)=w(\bar{u})$ for all $u \in U_{K^{\mathrm{alg}}}$, and $\mathbf{v}_{0}(c)=\left(0, v_{0}(c)\right)$ for all $c \in k$. The value group of $\left.\mathbf{v}_{0}\right|_{K^{*}}$ is isomorphic to $\mathbb{Z} \times \mathbb{Z}$.

Proof. Let $\pi_{0}$ be a prime element of $k_{0}$. For $a \in K^{\text {alg }}$, let $v_{0}(a)=\frac{m}{n}, m, n \in$ $\mathbb{Z}$. Then $\pi_{0}^{-m} a^{n} \in U_{K^{\text {alg }}}$, and we see that the only possible value for $\mathbf{v}_{0}(a)$ is $\left(w\left(\overline{\pi_{0}^{-m} a^{n}}\right) / n, m / n\right)$. The value group of $\left.\mathbf{v}_{0}\right|_{K^{*}}$ is generated by $\mathbf{v}_{0}(\pi)$ and $(1,0)$, where $\pi$ is a prime of $K$.

Introduce the index set

$$
\mathbb{I}_{2}=\mathbb{I} \cup\left\{\left(i_{1}, i_{2}\right) \mid i_{1}, i_{2} \in \mathbb{Q}, i_{2}>0\right\} .
$$

We extend the ordering of $\mathbb{I}$ onto $\mathbb{I}_{2}$ assuming

$$
\left(\mathbf{i}, i_{2}\right)<\left(i_{1}, i_{2}\right)<\left(i_{1}^{\prime}, i_{2}\right)<\left(\mathbf{i}, i_{2}^{\prime}\right)
$$

for all $i_{2}<i_{2}^{\prime}, i_{1}<i_{1}^{\prime}$. 
We turn to the definition of $G_{i_{1}, i_{2}}$ where $G$ is the Galois group of a given finite Galois extension $L / K$. Assume first that $K_{\mathbf{c}}$ is standard and $L / K_{\mathbf{c}}$ is ferociously ramified. Let $t \in \mathcal{O}_{L}, \bar{t} \notin \bar{L}^{p}$. We define

$$
G_{i_{1}, i_{2}}=\left\{g \in \operatorname{Gal}\left(L / K_{\mathbf{c}}\right) \mid \mathbf{v}_{0}\left(\frac{g(t)}{t}-1\right) \geq\left(i_{1}, i_{2}\right)\right\}
$$

for $i_{1}, i_{2} \in \mathbb{Q}, i_{2}>0$. Observe that $\mathcal{O}_{L}=\mathcal{O}_{K_{\mathrm{c}}}[t]$, whence $\mathbf{v}_{0}\left(g(t) t^{-1}-1\right) \geq$ $\left(i_{1}, i_{2}\right)$ implies $\mathbf{v}_{0}\left(g(a) a^{-1}-1\right) \geq\left(i_{1}, i_{2}\right)$ for any $a \in \mathcal{O}_{L}, a \neq 0$. This proves the independence of $G_{i_{1}, i_{2}}$ from the choice of $t$.

In the general case we choose $l^{\prime} / l$ such that $l^{\prime} K_{\mathbf{c}}$ is standard and $l^{\prime} L / l^{\prime} K_{\mathbf{c}}$ is ferociously ramified and put

$$
G_{i_{1}, i_{2}}=\operatorname{Gal}\left(l^{\prime} L / l^{\prime} K_{\mathbf{c}}\right)_{i_{1}, i_{2}} .
$$

Like in $\S 1$, we see that $G_{i_{1}, i_{2}}$ does not depend on the choice of $l^{\prime}$. (Therefore, only $k_{0}$ and $w$ are involved.)

We say that $\alpha \in \mathbb{I}_{2}$ is a jump for $L / K$ if $G_{\alpha} \neq G_{\beta}$ for any $\beta>\alpha$. It is easy to see that any jump is either -1 , or 0 , or $(\mathbf{c}, i)$ with integral $i$, or $\left(i_{1}, i_{2}\right)$.

Like in 1.3, one constructs Hasse-Herbrand functions $\Phi_{2, L / K}: \mathbb{I}_{2} \rightarrow \mathbb{I}_{2}$ and $\Psi_{2, L / K}=\Phi_{2, L / K}^{-1}$ which extend $\Phi$ and $\Psi$ respectively. Namely,

$$
\begin{aligned}
\Phi_{2, L / K}\left(\left(i_{1}, i_{2}\right)\right) & =\int_{(0,0)}^{\left(i_{1}, i_{2}\right)}\left|\operatorname{Gal}(L / K)_{t}\right| d t \\
& =\sum_{i=1}^{m}\left(h_{i}-h_{i-1}\right)\left|\operatorname{Gal}(L / K)_{h_{i}}\right|+\left(\left(i_{1}, i_{2}\right)-h_{m}\right)\left|\operatorname{Gal}(L / K)_{i_{1}, i_{2}}\right|
\end{aligned}
$$

where $h_{1}<\cdots<h_{m}$ are all jumps for $L / K$ between $(0,0)$ and $\left(i_{1}, i_{2}\right)$, and $h_{0}=$ $(0,0)$.

The assertions of $\mathbf{1 . 2}, \mathbf{1 . 3}, \mathbf{1 . 4}$ all remain valid, if one replaces $\mathbb{I}, \Phi, \Psi$ with $\mathbb{I}_{2}$, $\Phi_{2}, \Psi_{2}$ respectively. In what follows, we shall write $\Phi$ and $\Psi$ instead of $\Phi_{2}$ and $\Psi_{2}$.

\section{$\S 4$. The subgroups $S_{\alpha}$ IN $U(1) K_{2}^{\text {top }} K$}

In the rest of this paper $K$ denotes a two-dimensional local field (see $\mathbf{0 . 3}$ ) such that char $K=p$. We fix a base subfield in $K$. The constant subfield of $K$ is denoted by $k$.

We fix a diskrete valuation of renk one on the first residue field of $K$. It has a unique extension to the valuation $w:\left(\bar{K}^{\text {alg }}\right)^{*} \rightarrow \mathbb{Q}$. We do not require that $w\left(\bar{K}^{*}\right) \subset \mathbb{Z}$. Then a valuation $\mathbf{v}_{0}=\left(v_{1}, v_{2}\right):\left(K^{\text {alg }}\right)^{*} \rightarrow \mathbb{Q}^{2}$ is defined, see $\S 3$. Let $t, \pi$ be local parameters of $K$. Introduce a matrix

$$
\mathbf{e}_{\pi, t}=\left(\begin{array}{cc}
v_{1}(t) & v_{2}(t) \\
v_{1}(\pi) & v_{2}(\pi)
\end{array}\right)^{-1}
$$

Then it is easy to see that the valuation $\mathbf{v}_{K}: K^{*} \rightarrow \mathbb{Z}^{2}$, which is associated with $\pi$ and $t$, can be written as $\mathbf{v}_{K}=\left.\mathbf{v}_{0}\right|_{K} \cdot \mathbf{e}_{\pi, t}$. In most cases, we shall not mention explicitly that we have fixed certain local parameters $t, \pi$ in $K$. 
Sometimes we shall use the notation $U_{\alpha, K}$ for $\left\{u \in K \mid \mathbf{v}_{0}(u-1) \geq \alpha\right\}$.

Introduce subgroups

$$
\begin{aligned}
& Q_{\alpha}=Q_{\alpha, K}=\left\langle\{c, u\} \mid c \in k, u \in K, \mathbf{v}_{0}(u-1) \geq \alpha\right\rangle \subset U(1) K_{2}^{\mathrm{top}} K, \\
& Q_{\alpha}^{(n)}=\left\{a \in U(1) K_{2}^{\mathrm{top}} K \mid p^{n} a \in Q_{\alpha}\right\}, \\
& S_{\alpha}=S_{\alpha, K}=\mathrm{Cl} \bigcup_{n \geq 0} Q_{p^{n} \alpha}^{(n)},
\end{aligned}
$$

where $\alpha \in \mathbb{Q}_{+}^{2}, n \geq 0$.

To check that $S_{\alpha}$ is a subgroup in $U(1) K_{2}^{\text {top }} K$, observe that $p Q_{\alpha} \subset Q_{p \alpha}$. Therefore, $Q_{\alpha}^{(n)} \subset Q_{p \alpha}^{(n+1)}$ for all $n$.

Proposition 4.1. Let $K$ be standard, $\pi, t$ local parameters of $K$ such that $\pi$ is a constant. Then the following elements of $U(1) K_{2}^{\text {top }} K$ topologically generate $S_{\alpha}$ :

$$
\begin{aligned}
& p^{r_{i j}}\left\{\pi, 1+\theta \pi^{i} t^{j}\right\}, \quad \theta \in B,(j, p)=1 \\
& p^{r_{i j}}\left\{t, 1+\theta \pi^{i} t^{j}\right\}, \quad \theta \in B,(i, p)=1,(j, p) \neq 1, j \neq 0,
\end{aligned}
$$

where $B$ is a certain fixed $\mathbb{F}_{p}$-basis of the last residue field of $K, r_{i j}=r_{i j}(\alpha)$ is the minimal non-negative integer such that $p^{r_{i j}}(j, i) \geq p^{v_{p}(j)} \alpha \mathbf{e}_{\pi, t}$.

Corollary 1. In the setting of Proposition 4.1, the subgroup $p S_{\alpha / p}$, together with the following elements of $U(1) K_{2}^{\text {top }} K$, topologically generate $S_{\alpha}$ :

$$
\begin{aligned}
& \left\{\pi, 1+\theta \pi^{i} t^{j}\right\}, \quad \theta \in B, p \nmid j,(j, i) \geq \alpha \mathbf{e}_{\pi, t} ; \\
& \left\{t, 1+\theta \pi^{i} t^{j}\right\}, \quad \theta \in B, p \nmid i, p \mid j,(j, i) \geq p^{v_{p}(j)} \alpha \mathbf{e}_{\pi, t} .
\end{aligned}
$$

(One can say that these elements are topological generators of $S_{\alpha}$ modulo $p S_{\alpha / p}$.)

Corollary 2. In the setting of Proposition 4.1, let

$$
a_{i j \theta}= \begin{cases}\left\{\pi, u_{i j \theta}\right\}, & \theta \in B, p \nmid j,(j, i) \geq \alpha \mathbf{e}_{\pi, t} ; \\ \left\{t, u_{i j \theta}\right\}, & \theta \in B, p \nmid i, p \mid j,(j, i) \geq p^{v_{p}(j)} \alpha \mathbf{e}_{\pi, t} .\end{cases}
$$

where $u_{i j \theta} \equiv 1+\theta \pi^{i} t^{j} \bmod U_{K}(j+1, i)$. Assume that all $a_{i j \theta} \in S_{\alpha}$ and that $u_{i j \theta}$ form a part of some system of topological generators of $K^{*}$. Then all these $a_{i j \theta}$ are topological generators of $S_{\alpha}$ modulo $p S_{\alpha / p}$.

Next, for a fixed $t$, for any $j \neq 0$ and $i>0$ denote

$$
W_{i j}=W_{i j, K}=\left\{1+\sum_{\nu=1}^{\infty} c_{\nu} t^{\nu j} \mid c_{\nu} \in k, v_{k}\left(c_{\nu}\right) \geq \nu i\right\} .
$$

Obviously, $W_{i j}$ is a subgroup in $K^{*}$.

Proposition 4.2. Let $K=k\{\{t\}\}, \pi$ a prime element in $k, \alpha \in \mathbb{Q}_{+}^{2}, j \neq 0, i>0$, $(j, i) \geq p^{v_{p}(j)} \alpha \mathbf{e}_{\pi, t}$. Then for any $u \in W_{i j}$ we have $\{t, u\} \in S_{\alpha}$.

Proof of Propositions 4.1 and 4.2. Denote by $A_{\alpha}^{0}$ the subgroup of $U(1) K_{2}^{\text {top }} K$ generated by all elements (1) and put $A_{\alpha}=\mathrm{Cl} A_{\alpha}^{0}$. Denote also $B_{d}=\left\{\theta^{p^{d}} \mid \theta \in B\right\}$, $d \geq 0$. This is also an $\mathbb{F}_{p}$-basis of $F$. 
Step 1: $A_{\alpha} \subset S_{\alpha}$.

Let $(i, p)=1,(j, p) \neq 1$. Then

$$
\begin{aligned}
\left\{\pi, 1+\theta \pi^{i} t^{j}\right\} & =i^{-1}\left\{\pi^{i}, 1+\theta \pi^{i} t^{j}\right\} \\
& =i^{-1}\left\{-\theta t^{j}, 1+\theta \pi^{i} t^{j}\right\} \\
& =i^{-1} j\left\{t, 1+\theta \pi^{i} t^{j}\right\},
\end{aligned}
$$

whence $p^{v_{p}(j)}\left\{t, 1+\theta \pi^{i} t^{j}\right\} \in Q_{\beta}, \beta=(j, i) \mathbf{e}_{\pi, t}^{-1}$. If $r_{i j} \leq v_{p}(j)$, we have

$$
p^{r_{i j}}\left\{t, 1+\theta \pi^{i} t^{j}\right\} \in Q_{\beta}^{\left(v_{p}(j)-r_{i j}\right)} \subset S_{p^{r_{i j}-v_{p}(j)} \beta} \subset S_{\alpha}
$$

If $r_{i j}>v_{p}(j)$, we have

$$
p^{r_{i j}}\left\{t, 1+\theta \pi^{i} t^{j}\right\} \in p^{r_{i j}-v_{p}(j)} Q_{\beta} \subset Q_{p^{r_{i j}-v_{p}(j)} \beta} \subset S_{\alpha} .
$$

Similarly, for $(j, p)=1$ we have

$$
p^{r_{i j}}\left\{\pi, 1+\theta \pi^{i} t^{j}\right\} \in p^{r_{i j}} Q_{\beta} \subset Q_{p^{r_{i j} \beta}} \subset S_{\alpha} .
$$

Therefore, $A_{\alpha}^{0} \subset S_{\alpha}$, and $A_{\alpha} \subset S_{\alpha}$.

Step 2: the elements $p^{r_{i j}}\left\{\pi, 1+\theta \pi^{i} t^{j}\right\}$ and $p^{r_{i j}}\left\{t, 1+\theta \pi^{i} t^{j}\right\}$ are in $A_{\alpha}$ for all $i>0$, all $j \neq 0$, and all $\theta \in B_{d}, d=\min \left(v_{p}(i), v_{p}(j)\right)$.

If $(i, p)=1,(j, p) \neq 1$, the symbol $p^{r_{i j}}\left\{\pi, 1+\theta \pi^{i} t^{j}\right\}$ is a multiple of $p^{r_{i j}}\{t, 1+$ $\left.\theta \pi^{i} t^{j}\right\}$, see (2). The case $(j, p)=1$ is similar. If $d=\min \left(v_{p}(i), v_{p}(j)\right)>0, i=p^{d} i_{0}$ and $j=p^{d} j_{0}$, we have $r_{i_{0} j_{0}}=r_{i j}$, and for $\tau=\pi, t$ :

$$
p^{r_{i j}}\left\{\tau, 1+\theta \pi^{i} t^{j}\right\}=p^{d} \cdot p^{r_{i_{0} j_{0}}}\left\{\tau, 1+\theta_{0} \pi^{i_{0}} t^{j_{0}}\right\} \in A_{\alpha} .
$$

Step 3: elements $\{t, u\}$ (from Prop. 4.2) belong to $A_{\alpha}$.

Any element of $W_{i j}$ can be written as an infinite product of $1+\theta \pi^{m} t^{n j}$ with $m \geq n i>0$ and $\theta \in B_{d}, d=\min \left(v_{p}(m), v_{p}(n i)\right)$. We have

$$
(n j, m) \geq(n j, n i) \geq n p^{v_{p}(j)} \alpha \mathbf{e}_{\pi, t} \geq p^{v_{p}(n)} p^{v_{p}(j)} \alpha \mathbf{e}_{\pi, t}=p^{v_{p}(n j)} \alpha \mathbf{e}_{\pi, t} .
$$

Therefore, $r_{m, n j}=0$, whence $\left\{t, 1+\theta \pi^{m} t^{n j}\right\}$ are in $A_{\alpha}$ by Step 2 .

Step 4: $Q_{\alpha} \subset A_{\alpha}$ (for all al) implies $S_{\alpha} \subset A_{\alpha}$.

Indeed, $A_{\alpha}$ consists of all $a \in U(1) K_{2}^{\text {top }} K$ such that in the standard expansion of $a$ as in Proposition 0.4.5 we have $v_{p}\left(c_{i j \theta}\right) \geq r_{i j}(\alpha)$ whenever $j \neq 0$, and $c_{i j \theta}=0$ when $j=0$. Since $\max \left(r_{i j}\left(p^{n} \alpha\right), n\right)=r_{i j}(\alpha)+n$, we obtain

$$
A_{p^{n} \alpha} \cap p^{n} U(1) K_{2}^{\mathrm{top}} K=p^{n} A_{\alpha} .
$$

Therefore, $Q_{\alpha} \subset A_{\alpha}$ implies $Q_{p^{n} \alpha}^{(n)} \subset A_{\alpha}$.

Step 5: $Q_{\alpha} \subset A_{\alpha}$.

The group $Q_{\alpha}$ is topologically generated by $\left\{\pi, 1+\theta \pi^{i} t^{j}\right\}$ and $\left\{1+\eta \pi^{r}, 1+\theta \pi^{i} t^{j}\right\}$, where $\theta, \eta \in B,(j, i) \geq \alpha \mathbf{e}_{\pi, t}$, and $r$ is any positive integer. We have to check that these elements are in $A_{\alpha}$. 
If $j=0$, then all these elements are equal to 0 since $K_{2}^{\text {top }} k=0$ (see $[\mathrm{Kn}$, Théorème 1]). Thus, we may assume $j \neq 0$. We may also reduce to the case $p \nmid(i, j)$. Indeed, if $\min \left(v_{p}(i), v_{p}(j)\right)=d>0$, we have

$$
\left\{\pi, 1+\theta \pi^{i} t^{j}\right\}=p^{d}\left\{\pi, 1+\theta^{p^{-d}} \pi^{i_{0}} t^{j_{0}}\right\} \in p^{d} A_{\alpha / p^{d}} \subset A_{\alpha},
$$

and the same for $\left\{1+\eta \pi^{r}, 1+\theta \pi^{i} t^{j}\right\}$.

If $(j, p)=1$, we have $r_{j i}(\alpha)=0$, and $\left\{\pi, 1+\theta \pi^{i} t^{j}\right\}$ is just one of the elements (1). If $(j, p) \neq 1$, we have $r_{j i}(\alpha) \leq v_{p}(j)$ and, by $(2),\left\{\pi, 1+\theta \pi^{i} t^{j}\right\}$ is a multiple of $p^{r_{i j}}\left\{t, 1+\theta \pi^{i} t^{j}\right\}$ which is one of the elements (1).

Finally, by Lemma 0.4.1,

$$
\begin{aligned}
\left\{1+\eta \pi^{r}, 1+\theta \pi^{i} t^{j}\right\} & =-\left\{1+\theta \pi^{i} t^{j}, 1+\eta \pi^{r}\right\} \\
& =-\left\{-\theta \pi^{i} t^{j}\left(1+\eta \pi^{r}\right), w\right\}
\end{aligned}
$$

where

$$
w=1+\frac{\theta \eta \pi^{i+r} t^{j}}{1+\theta \pi^{i} t^{j}} \in W_{i j} \cap U_{K}(i+r) .
$$

The symbol $\{-\theta, w\}$ is infinitely divisible, and, therefore, equals 0 in $K_{2}^{\text {top }} K$. We conclude that

$$
\left\{1+\eta \pi^{r}, 1+\theta \pi^{i} t^{j}\right\}=-i\{\pi, w\}-j\{t, w\}-\left\{1+\eta \pi^{r}, w\right\}
$$

We already know from the previous paragraph that $\{\pi, w\} \in A_{\alpha}$. Next, $j\{t, w\}$ is a multiple of $p^{v_{p}(j)}\{t, w\}$, and $\{t, w\} \in A_{\alpha / p^{v_{p}(j)}}$ by Step 3. Therefore, $j\{t, w\} \in$ $p^{v_{p}(j)} A_{\alpha / p^{v_{p}(j)}} \subset A_{\alpha}$. Applying the same transformation (3) to $\left\{1+\eta \pi^{r}, w\right\}$, we expand $\left\{1+\eta \pi^{r}, 1+\theta \pi^{i} t^{j}\right\}$ into a convergent sum of elements of $A_{\alpha}$.

\section{$\S 5$. Behavior of $S_{\alpha}$ In CERTAin types of EXTENSIONS}

5.1. In this section we assume that $K$ is an equal characteristic two-dimensional local field with the following additional property.

(*) Let $L / K$ be any finite unramified extension. Then the extension $l / k$ is also unramified, where $k$ and $l$ are the constant subfields of $K$ and $L$ respectively.

The class of such fields is stable with respect to constant or unramified extensions. However, an almost constant extension of $K$ need not be of this type: adjoin a root of $X^{p}-X-\pi^{-1}-t$ to $\mathbb{F}_{p}((\pi))\{\{t\}\}$. Note that any standard field satisfies $\left(^{*}\right)$.

This condition on $K$, together with Proposition 0.2.1, easily implies that a certain constant purely inseparable extension of $K$ is standard.

The aim of this section is to prove the following three assertions.

Proposition 5.1.1. Let $L / K$ be an unramified extension, and let $\alpha \in \mathbb{Q}_{+}^{2}$. Then we have $N_{L / K} S_{\alpha, L}=S_{\alpha, K}$.

Proposition 5.1.2. Let $L / K$ be a constant totally ramified extension, and let $\alpha \in \mathbb{Q}_{+}^{2}$. Then $N_{L / K} S_{\alpha, L}=S_{\alpha, K}$. 
Proposition 5.1.3. Let $K$ be standard, $L / K$ a cyclic ferociously ramified extension of degree $p$ with the ramification jump $h$ in lower numbering, and let $\alpha \in \mathbb{Q}_{+}^{2}$. Then:

(1) $N_{L / K} S_{\alpha, L}=S_{\alpha+(p-1) h, K}$ if $\alpha>h$;

(2) $N_{L / K} S_{\alpha, L}$ is a subgroup in $S_{p \alpha, K}$ if $\alpha \leq h$;

(3) $\left(S_{p \alpha, K}: N_{L / K} S_{\alpha, L}\right)=p$ if $\alpha \leq h$ and the last residue field of $K$ is quasifinite.

For the purposes of the proof, it is convenient to introduce notation $S_{\alpha, K}^{0}=$ $\bigcup_{n>0} Q_{p^{n} \alpha}^{(n)}$. Then $S_{\alpha, K}=\mathrm{Cl} S_{\alpha, K}^{0}$, and to prove, say, Proposition 5.1.1, it is sufficient to check that its conditions imply that $N_{L / K} S_{\alpha, L}^{0}$ is dense in $S_{\alpha, K}$.

5.2. Let $L / K$ be a finite extension. Since there is no torsion in $V K_{2}^{\text {top }} K$, the natural map $V K_{2}^{\text {top }} K \rightarrow V K_{2}^{\text {top }} L$ is injective, and we may identify $V K_{2}^{\text {top }} K$ with a subgroup in $V K_{2}^{\text {top }} L$.

Lemma 1. Let $L / K$ be inseparable of degree $p$. Then $V K_{2}^{\mathrm{top}} K \subset p V K_{2}^{\mathrm{top}} L$.

Proof. Let $L / K$ be totally ramified. Let $\pi, t$ be local parameters of $L$. Then $\pi^{p}, t$ are local parameters of $K$. In $V K_{2}^{\text {top }} K$ one can choose a system of topological generators of type $\left\{\pi^{p}, 1+\theta\left(\pi^{p}\right)^{i} t^{j}\right\}, p \nmid j$, and $\left\{t, 1+\theta\left(\pi^{p}\right)^{i} t^{j}\right\}, p \mid j$. Evidently, all these generators lie in $p V K_{2}^{\text {top }} L$.

For a ferociously ramified $L / K$, one should change the roles of $\pi$ and $t$.

Lemma 2. Let $L / K$ be an inseparable constant extension of degree $p$. Then $S_{\alpha, K}^{0} \subset$ $S_{p \alpha, L}^{0}$.

Proof. It is sufficient to check that $Q_{\alpha, K} \subset Q_{p \alpha, L}$. Let $l$ and $k$ be the constant subfields in $L$ and $K$ respectively. Then $k=l^{p}$,

$$
\begin{aligned}
Q_{\alpha, K} & \left.\left.=\left\langle\left\{c^{p}, u\right\}\right| c \in l, u \in K, \mathbf{v}_{0}(u-1) \geq \alpha\right\}\right\rangle \\
& \left.\left.\subset\left\langle\left\{c^{p}, u\right\}\right| c \in l, u \in L, \mathbf{v}_{0}(u-1) \geq \alpha\right\}\right\rangle \\
& \left.\left.=\left\langle\left\{c, u^{p}\right\}\right| c \in l, u \in L, \mathbf{v}_{0}(u-1) \geq \alpha\right\}\right\rangle \\
& \left.\left.\subset\langle\{c, u\}| c \in l, u \in L, \mathbf{v}_{0}(u-1) \geq p \alpha\right\}\right\rangle \\
& =Q_{p \alpha, L} .
\end{aligned}
$$

Proposition 5.2. Let $L / K$ be a constant purely inseparable totally ramified extension, and let $\alpha \in \mathbb{Q}_{+}^{2}$. Then $N_{L / K} S_{\alpha, L}=S_{\alpha, K}$.

Proof. We may assume $[L: K]=p$. Let $\pi$ be a prime in the constant subfield of $L$. We have

$$
\begin{aligned}
N_{L / K}\left(p Q_{\alpha, L}\right) & \left.\left.=N_{L / K}\left(\left\langle\left\{c^{p}, u\right\}\right| c \in l, u \in L, \mathbf{v}_{0}(u-1) \geq \alpha\right\}\right\rangle\right) \\
& \left.\left.=\left\langle\left\{c^{p}, u^{p}\right\}\right| c \in l, u \in L, \mathbf{v}_{0}(u-1) \geq \alpha\right\}\right\rangle \\
& \left.\left.\subset\left\langle\left\{c^{p}, u\right\}\right| c \in l, u \in K, \mathbf{v}_{0}(u-1) \geq p \alpha\right\}\right\rangle \\
& =Q_{p \alpha, K},
\end{aligned}
$$

whence $N_{L / K} Q_{\alpha, L} \subset S_{\alpha, K}$, and $N_{L / K} S_{\alpha, L} \subset S_{\alpha, K}$ for all $\alpha \in \mathbb{Q}_{+}^{2}$. Conversely, let $a \in S_{\alpha, K}^{0}$. Then $a=p b=N_{L / K} b, b \in V K_{2}^{\text {top }} L$ by Lemma 1. By Lemma 2, $p b \in S_{p \alpha, L}^{0}$, whence $b \in S_{\alpha, L}^{0}$. 


\subsection{Proof of Proposition 5.1.1.}

By Proposition 0.2.1 and condition $(*)$, there exists an inseparable constant extension $K^{\prime} / K$ such that $K^{\prime}$ is standard. In view of Proposition 5.2, we may assume without loss of generality that $K^{\prime}=K=k\{\{t\}\}, L=l\{\{t\}\}$. Fix a prime $\pi \in k$, it is also a prime element of $l$.

To prove that $N_{L / K} S_{\alpha, L} \subset S_{\alpha, K}$, it is sufficient to check that the norms of all standard topological generators of $N_{L / K} S_{\alpha, L}$ (as in Prop. 4.1) belong to $S_{\alpha, K}$. Since $p^{r} S_{\alpha / p^{r}, K} \subset S_{\alpha, K}$, we may consider only the case $r_{i j}=0$. Now, for $(j, i) \geq$ $\alpha \mathbf{e}_{\pi, t}$,

$$
N_{L / K}\left\{\pi, 1+\theta \pi^{i} t^{j}\right\}=\left\{\pi, N_{L / K}\left(1+\theta \pi^{i} t^{j}\right)\right\} \in Q_{\alpha, K} \subset S_{\alpha, K}
$$

by the definition of $Q_{\alpha, K}$. Next, for $(j, i) \geq p^{v_{p}(j)} \alpha \mathbf{e}_{\pi, t}$,

$$
N_{L / K}\left\{t, 1+\theta \pi^{i} t^{j}\right\}=\left\{t, N_{L / K}\left(1+\theta \pi^{i} t^{j}\right)\right\} \in S_{\alpha, K}
$$

Indeed, all the conjugates of $1+\theta \pi^{i} t^{j}$ are in $W_{i j, L}$, whence $N_{L / K}\left(1+\theta \pi^{i} t^{j}\right) \in$ $W_{i j, L} \cap K=W_{i j, K}$, and we may apply Prop. 4.2.

Conversely, to prove that $S_{\alpha, K} \subset N_{L / K} S_{\alpha, L}$, it is sufficient to check that all standard topological generators of $S_{\alpha, K}$ belong to $N_{L / K} S_{\alpha, L}$ (see Prop. 0.4.4). Again, we can easily reduce to the case $r_{i j}=0$. We have $1+\theta \pi^{i} t^{j} \in N_{L / K} U_{L}(j, i)$, whence, for $(j, i) \geq \alpha \mathbf{e}_{\pi, t}$,

$$
\left\{\pi, 1+\theta \pi^{i} t^{j}\right\} \in N_{L / K} Q_{\alpha, L} \subset N_{L / K} S_{\alpha, L}
$$

To see that $\left\{t, 1+\theta \pi^{i} t^{j}\right\} \in N_{L / K} S_{\alpha, L}$ for $(j, i) \geq p^{v_{p}(j)} \alpha \mathbf{e}_{\pi, t}$, observe that the norms of topological generators of $W_{i j, L}$ topologically generate $W_{i j, K}$, whence $N_{L / K} W_{i j, L}=W_{i j, K}$.

\subsection{Proof of Proposition 5.1.2.}

Let $L / K$ be a constant totally ramified extension. Denote by $\tilde{L} / K$ the normal closure of $L / K$, by $K^{\prime} / K$ the maximal unramified subextension in $\tilde{L} / K$ and by $L^{\prime} / L$ that in $\tilde{L} / L$. By Proposition 5.1.1, $S_{\alpha, L}=N_{L^{\prime} / L} S_{\alpha, L^{\prime}}$, and $S_{\alpha, K}=N_{K^{\prime} / K} S_{\alpha, K^{\prime}}$, and it is sufficient to prove Proposition for $\tilde{L} / L^{\prime}$ and $\tilde{L} / K^{\prime}$.

Therefore, we may assume that $L / K$ is normal, and, further, that $L / K$ is cyclic of prime degree. As in 5.3, there exists an inseparable constant extension $K^{\prime} / K$ such that $K^{\prime}$ is standard. In view of Proposition 5.2, we may assume without loss of generality that $K^{\prime}=K$.

Let $\pi, t$ be local parameters in $L$ such that $\pi$ is a constant. Then $\pi_{0}, t$ are local parameters of $K$, where $\pi_{0}=N_{L / K} \pi$; we have $K=k\{\{t\}\}$.

We start with the case $[L: K]=p$. Let the ramification jump of $L / K$ be $(\mathbf{c}, h)$.

First, we prove that $N_{L / K} S_{\alpha, L} \subset S_{\alpha, K}$. Notice that the group $\left\{\{\pi, u\} \mid \mathbf{v}_{0}(u-\right.$ 1) $\geq \alpha\}$ is topologically generated by

$$
\begin{aligned}
& \left\{\pi, 1+\theta \pi_{0}^{i} t^{j}\right\}, \quad(j, p i) \geq \alpha \mathbf{e}_{\pi, t}, \\
& \left\{\pi, 1+\theta \pi^{i} t^{j}\right\}, \quad(i, p)=1,(j, i) \geq \alpha \mathbf{e}_{\pi, t} .
\end{aligned}
$$

It follows from Prop. 4.1 that $S_{\alpha, L}$ is topologically generated by these elements and all $p^{r_{i j}}\left\{t, 1+\theta \pi^{i} t^{j}\right\}$ of Prop. 4.1. As in 5.3, it is sufficient to consider only those $(j, i)$ where $r_{i j}=0$. Notice also that $(i, p)=1$ implies that $\left\{\pi, 1+\theta \pi^{i} t^{j}\right\}$ is a multiple of $\left\{t, 1+\theta \pi^{i} t^{j}\right\}$ (see (2) in $\left.\S 4\right)$. 
Therefore, $N_{L / K} Q_{\alpha, L}$ is topologically generated by

$$
\left.N_{L / K}\left\{\pi, 1+\theta \pi_{0}^{i} t^{j}\right\}=\left\{\pi_{0}, 1+\theta \pi_{0}^{i} t^{j}\right\}, \quad(j, p i) \geq \alpha \mathbf{e}_{\pi, t} \text { (i. e., }(j, i) \geq \alpha \mathbf{e}_{\pi_{0}, t}\right),
$$

and

$$
\begin{aligned}
N_{L / K}\left\{t, 1+\theta \pi^{i} t^{j}\right\} & =\left\{t, N_{L / K}\left(1+\theta \pi^{i} t^{j}\right)\right\} \\
& =\left\{t, 1+\sum_{\nu=1}^{p} c_{\nu} t^{\nu j}\right\}, \quad(i, p)=1,(j, i) \geq v_{p}(j) \alpha \mathbf{e}_{\pi, t} .
\end{aligned}
$$

Here $c_{\nu} \in k, v_{k}\left(c_{\nu}\right) \geq \frac{1}{p}(\nu i+(p-1) h), \nu=1, \ldots, p-1 ; v_{k}\left(c_{p}\right)=i$. Then Proposition 4.2 implies that $N_{L / K}\left\{t, 1+\theta \pi^{i} t^{j}\right\} \in S_{\alpha, K}$ (note that $(j, i / p) \geq v_{p}(j) \alpha \mathbf{e}_{\pi_{0}, t}$ ).

It remains to prove that $N_{L / K} S_{\alpha, L}$ is dense in $S_{\alpha, K}$. In view of Proposition 0.4.3, it is sufficient to show that $N_{L / K} S_{\alpha, L}+p S_{\alpha / p, K}$ is dense in $S_{\alpha, K}$. To do this, we shall construct a system of topological generators $a_{i j \theta}$ for $S_{\alpha, K} / p S_{\alpha / p, K}$ as in Corollary 2 to Proposition 4.1 such that $a_{i j \theta} \in N_{L / K} S_{\alpha, L}$. In this argument we shall also make additional requirement for all $a_{i j \theta}$ with $v_{p}(i)=0$. Let $j=p^{m} j_{0}$, $m=v_{p}(j)$. We require that $a_{i j \theta}=\left\{t, u_{i j \theta}\right\}$, where

$$
u_{i j \theta}=1+\sum_{\nu=p^{m}}^{\infty} c_{\nu} t^{\nu j_{0}}
$$

with $c_{\nu} \in k, v_{k}\left(c_{\nu}\right) \geq \frac{i}{p^{m}} \nu$.

Apply induction on $v_{p}(j)$. If $v_{p}(j)=0$, take $a_{i j \theta}=N_{L / K}\left\{\pi, 1+\theta \pi_{0}^{i} t^{j}\right\}$ for all $(j, i) \geq \alpha \mathbf{e}_{\pi_{0}, t}$.

Suppose that $a_{i j \theta}$ with $v_{p}(j) \leq n$ have already been constructed. Take a pair $(j, i)$ with $v_{p}(j)=n+1$ and $(j, i) \geq p^{n+1} \alpha \mathbf{e}_{\pi_{0}, t}$. Consider

$$
\begin{aligned}
N_{L / K}\left\{t, 1+\theta \pi^{i} t^{j / p}\right\} & =\left\{t, N_{L / K}\left(1+\theta \pi^{i} t^{j / p}\right)\right\} \\
& =\left\{t, 1+\sum_{r=1}^{p-1} c_{r} t^{r j / p}+\theta^{p} \pi_{0}^{i} t^{j}\right\},
\end{aligned}
$$

where $c_{r} \in k, v_{k}\left(c_{r}\right) \geq r i+(p-1) h$. We take $a_{i j \eta^{p}}=N_{L / K}\left\{t, 1+\eta \pi^{i} t^{j / p}\right\}$ if $(j, i)<(0, h)$. In the remaining case it is easy to see that for some integers $m_{r s \theta}$, $r=1, \ldots, p-1, s \geq v_{k}\left(c_{r}\right), \theta \in B$ we have

$$
N_{L / K}\left(1+\eta \pi^{i} t^{j / p}\right) \prod_{r, s, \theta} u_{r j / p, s, \theta}^{m_{r s \theta}} \equiv 1+\eta^{p} \pi_{0}^{i} t^{j} \quad \bmod U_{j+1, i, K}
$$

The left-hand side of this relation can be then taken as $u_{i j \eta^{p}}$. It is seen immediately that it satisfies (1), and we put $a_{i j \eta^{p}}=\left\{t, u_{i j \eta^{p}}\right\}$.

Then all these $a_{i j \theta}$ belong to $N_{L / K} S_{\alpha, L}$, and they topologically generate the quotient group $S_{\alpha, K} / p S_{\alpha / p, K}$ by Corollary 2 to Proposition 4.1.

It remains to consider the case $[L: K]=q \neq p$. Since $Q_{\alpha, K}=q Q_{\alpha, K}$, we have

$$
S_{\alpha, K}=q S_{\alpha, K}=N_{L / K} S_{\alpha, K} \subset N_{L / K} S_{\alpha, L}
$$

The converse can be proved as in $\mathbf{5 . 3}$. 


\subsection{Proof of Proposition 5.1.3.}

Let $\pi$ and $t$ be local parameters in $L$. Then $\pi$ and $t_{0}=N_{L / K} t$ are local parameters in $K$.

First, we prove that $N_{L / K} S_{\alpha, L} \subset S_{p \alpha, K}$ for $\alpha \leq h$. It is sufficient to check that $N_{L / K}\left(S_{\alpha, L} / p S_{\alpha / p, L}\right) \subset S_{p \alpha, K} / p S_{\alpha, K}$. The quotient group $S_{\alpha, L} / p S_{\alpha / p, L}$ is generated by the classes of $N_{L / K}\left\{\pi, 1+\theta \pi^{i} t^{j}\right\},(j, i) \geq \alpha \mathbf{e}_{\pi, t}$, and $N_{L / K}\{t, 1+$ $\left.\theta \pi^{i} t_{0}^{j}\right\},(j, i) \geq p^{v_{p}(j)+1} \alpha \mathbf{e}_{\pi, t}$, use Proposition 4.2. We have

$$
\begin{aligned}
N_{L / K}\left\{\pi, 1+\theta \pi^{i} t^{j}\right\} & =\left\{\pi, N_{L / K}\left(1+\theta \pi^{i} t^{j}\right)\right\} \in S_{p \alpha, K}, \\
N_{L / K}\left\{t, 1+\theta \pi^{i} t_{0}^{j}\right\} & =\left\{t_{0}, 1+\theta \pi^{i} t_{0}^{j}\right\} \in S_{p \alpha, K} .
\end{aligned}
$$

Similarly one shows $N_{L / K} S_{\alpha, L} \subset S_{\alpha+(p-1) h}$ for $\alpha \geq h$.

Next, we have $N_{L / K} Q_{\alpha, L}=Q_{\alpha+(p-1) h, K}$ for all $\alpha>h$. Let $n>0$. Denote $K_{n}=k\left\{\left\{t_{0}^{p^{n}}\right\}\right\} \subset K, L_{n}=k\left\{\left\{t^{p^{n}}\right\}\right\} \subset L$, where $k$ is the constant subfield of $K$. Then $L_{n} / K_{n}$ is a ferociously ramified extension with the ramification jump $p^{n} h$. It follows

$$
p^{n} N_{L / K} Q_{p^{n} \alpha, L}^{(n)}=N_{L_{n} / K_{n}} Q_{p^{n} \alpha, L_{n}}=Q_{p^{n} \alpha+(p-1) p^{n} h, K_{n}}=p^{n} Q_{p^{n} \alpha+p^{n}(p-1) h, K}^{(n)},
$$

whence $N_{L / K} Q_{p^{n} \alpha, L}^{(n)}=Q_{p^{n} \alpha+p^{n}(p-1) h, K}^{(n)}$. Since all $Q_{p^{n} \alpha, L}^{(n)}$ topologically generate $S_{\alpha, K}$, we conclude $N_{L / K} S_{\alpha, L}=S_{\alpha+(p-1) h, K}$ for all $\alpha>h$.

It remains to prove the third part of Proposition. Assume that the last residue field of $K$ is quasi-finite. Then the map

$$
N_{L / K}: U_{L}(j, i) / U_{L}(j+1, i) \rightarrow U_{K}(j, p i) / U_{K}(j+1, p i)
$$

has the cokernel of order $p$, where $(j, i)=\alpha \mathbf{e}_{\pi, t}$. Let $u \in U_{K}(j, p i)$ generate this cokernel. Then $\{\pi, u\} \notin N_{L / K} K_{2}^{\text {top }} L$, and $\{\pi, u\} \in Q_{h, K} \subset S_{\alpha, K}$ for any $\alpha \leq h$. This shows $\left(S_{p \alpha, K}: N_{L / K} S_{\alpha, L}\right) \geq p$ for $\alpha \leq h$.

Application of subfields $K_{n}$ and $L_{n}$ yields an easy calculation of all $N_{L / K}\{\pi, 1+$ $\left.\theta \pi^{i} t^{j}\right\}$ and $N_{L / K}\left\{t, 1+\theta \pi^{i} t^{j}\right\}$. We obtain that $N_{L / K} S_{\alpha, L}$ together with $\{\pi, u\}$ generates $S_{p \alpha, K}$ for all $\alpha \leq h$, whence $\left(S_{p \alpha, K}: N_{L / K} S_{\alpha, L}\right) \leq p$ for $\alpha \leq h$.

\section{§6. A filtration on $K_{2}^{\text {top }} K$ AND RECiPRocity MAP.}

In this section $K$ is a two-dimensional local field with a quasi-finite residue field, $\operatorname{char} K=\operatorname{char} \bar{K}=p$.

6.1. For any $\alpha \in \mathbb{I}_{2}$ we introduce a subgroup $F i l_{\alpha} K_{2}^{\text {top }} K \subset K_{2}^{\text {top }} K$ so that $F i l_{\beta} K_{2}^{\text {top }} K \subset F i l_{\alpha} K_{2}^{\text {top }} K$ whenever $\alpha<\beta$. It is easy to see that for some unramified extension $\tilde{K} / K$ the field $\tilde{K}$ satisfies the condition $(*)$, see $\S 5$. Denote

$F i l_{\infty} K_{2}^{\text {top }} K=0$;

$F i l_{\alpha} K_{2}^{\text {top }} K=N_{\tilde{K} / K} S_{\alpha, \tilde{K}}$ for $\alpha \in \mathbb{Q}_{+}^{2}$;

$F i l_{\mathbf{i}, \alpha_{2}} K_{2}^{\text {top }} K=\mathrm{Cl} \bigcup_{\alpha_{1} \in \mathbb{Q}} F i l_{\alpha_{1}, \alpha_{2}} K_{2}^{\text {top }} K$ for $\alpha_{2} \in \mathbb{Q}_{+}$;

$F i l_{\mathbf{c}, \infty} K_{2}^{\mathrm{top}} K=T_{K}:=\mathrm{Cl} \bigcup_{\alpha \in \mathbb{Q}_{+}^{2}} F i l_{\alpha} K_{2}^{\mathrm{top}} K$

$F i l_{\mathbf{c}, i} K_{2}^{\text {top }} K=T_{K}+\{\{t, u\} \mid u \in k, v(u-1) \geq i\}$ for all $i \in \mathbb{Q}_{+}$if $K=k\{\{t\}\}$ is standard; 
$F i l_{\mathbf{c}, i} K_{2}^{\mathrm{top}} K=N_{K^{\prime} / K} F i l_{\mathbf{c}, i} K_{2}^{\mathrm{top}} K^{\prime}$, if $K$ has the property $\left(^{*}\right)$ and $K^{\prime}$ is a purely inseparable constant extension such that $K^{\prime}$ is standard;

$F i l_{\mathbf{c}, i} K_{2}^{\text {top }} K=N_{\tilde{K} / K} F i l_{\mathbf{c}, i} K_{2}^{\text {top }} \tilde{K}$ in the general case, where $\tilde{K} / K$ is an unramified extension such that $\tilde{K}$ satisfies $\left(^{*}\right)$;

$F i l_{0} K_{2}^{\mathrm{top}} K=U(1) K_{2}^{\mathrm{top}} K+\left\{\{t, \theta\} \mid \theta \in \mathcal{R}_{K}\right\}$

$\mathrm{Fil}_{-1} K_{2}^{\mathrm{top}} K=K_{2}^{\mathrm{top}} K$.

Proposition 6.1.1. $F i l_{\alpha} K_{2}^{\mathrm{top}} K$ are well defined.

Proof. Independence from the choice of $\tilde{K}$ follows from Proposition 5.1.1 and from $N_{k^{\prime \prime} / k^{\prime}} U_{i, k^{\prime \prime}}=U_{i, k^{\prime}}$ in an unramified $k^{\prime \prime} / k^{\prime}$. If $u^{\prime} \in U_{K}, u \in U_{k}(1)$, then the definition of $Q_{\alpha}$ implies $\left\{u^{\prime}, u\right\} \in T_{K}$. This shows independence from the choice of $t$. Independence from the choice of $K^{\prime}$ follows from two observations:

(1) $N_{K^{\prime \prime} / K^{\prime}} T_{K^{\prime \prime}}=T_{K^{\prime}}$ if $K^{\prime \prime}=k^{\prime \prime}\{\{t\}\}, K^{\prime}=k^{\prime}\{\{t\}\}, k^{\prime \prime} / k^{\prime}$ is purely inseparable;

(2) in this case $N_{k^{\prime \prime} / k^{\prime}} U_{i, k^{\prime \prime}}=U_{i, k^{\prime}}$.

Proposition 6.1.2. $\bigcup_{\alpha>0} F i l_{\alpha} K_{2}^{\mathrm{top}} K=U(1) K_{2}^{\mathrm{top}} K$.

Proof. This is clear if $K$ is standard. Next, $N_{K^{\prime} / K} U(1) K_{2}^{\text {top }} K^{\prime}=U(1) K_{2}^{\text {top }} K$ if $K^{\prime} / K$ is a purely inseparable or unramified extension.

Remark. If $K$ is standard, then obviously

$$
F i l_{\mathbf{c}, i} K_{2}^{\mathrm{top}} K=T_{K}+U(i) K_{2}^{\mathrm{top}} K
$$

for a positive integer $i$.

\subsection{Norm map in a purely inseparable constant extension.}

Proposition 6.2. Let $L / K$ be a finite purely inseparable constant extension. Then $N_{L / K} F i l_{\alpha} K_{2}^{\text {top }} L=F i l_{\alpha} K_{2}^{\text {top }} K$ for all $\alpha \in \mathbb{I}_{2}$.

Proof. For $\alpha=\left(\mathbf{i}, \alpha_{2}\right)$, or $\alpha \in \mathbb{Q}_{+}^{2}$, see Proposition 5.1.2. The cases $\alpha=-1,(\mathbf{c}, \infty)$, $\infty$ are trivial. For $\alpha=(\mathbf{c}, i)$, it is sufficient to apply the observations in the proof of Proposition 6.1.1. Finally, let $\alpha=0$. We have

$$
N_{L / K} U(1) K_{2}^{\mathrm{top}} L=U(1) K_{2}^{\mathrm{top}} K
$$

and

$$
N_{L / K}(\{t, \theta\})=\left\{t, \theta^{p^{n}}\right\}
$$

for all $\theta \in \mathcal{R}_{K}=\mathcal{R}_{L}$, where $p^{n}=[L: K]$. Therefore,

$$
N_{L / K} F i l_{0} K_{2}^{\mathrm{top}} L=F i l_{0} K_{2}^{\mathrm{top}} K
$$

\subsection{Norm map in a cyclic extension of prime degree.}

Proposition 6.3. Let $L / K$ be a cyclic extension of prime degree, $G=\operatorname{Gal}(L / K)$, $h \in \mathbb{I}_{2}$ the only jump of ramification for $L / K, i$. e., $G_{h}=G$. Then:

1. If $\alpha>h$, then $N_{L / K} F i l_{\alpha} K_{2}^{\text {top }} L=F i l_{\Phi_{L / K}(\alpha)} K_{2}^{\text {top }} K$.

2. If $\alpha \leq h$, then $N_{L / K} F i l_{\alpha} K_{2}^{\text {top }} L$ is a subgroup in $F i l_{\Phi_{L / K}(\alpha)} K_{2}^{\text {top }} K$ of index $[L: K]$.

Proof. 
1. Reduction to the case when both $K$ and $L$ are almost standard. Choose purely inseparable $k^{\prime} / k$ such that $K^{\prime}=k^{\prime} K$ and $L^{\prime}=k^{\prime} L$ are almost standard. It is easy to see that $\Phi_{L^{\prime} / K^{\prime}}=\Phi_{L / K}$. Suppose Proposition is valid for $L^{\prime} / K^{\prime}$.

Let $\alpha>h$. Then

$$
\begin{aligned}
N_{L / K} F i l_{\alpha} K_{2}^{\mathrm{top}} L & =N_{L / K} N_{L^{\prime} / L} F i l_{\alpha} K_{2}^{\mathrm{top}} L^{\prime} \\
& =N_{K^{\prime} / K} F i l_{\Phi_{L^{\prime} / K^{\prime}}(\alpha)} K_{2}^{\mathrm{top}} K^{\prime} \\
& =N_{K^{\prime} / K} F i l_{\Phi_{L / K}(\alpha)} K_{2}^{\mathrm{top}} K^{\prime} \\
& =F i l_{\Phi_{L / K}(\alpha)} K_{2}^{\mathrm{top}} K .
\end{aligned}
$$

Let $\alpha \leq h$. Then

$$
F i l_{\Phi_{L^{\prime} / K^{\prime}}(\alpha)} K_{2}^{\mathrm{top}} K^{\prime} \supset N_{L^{\prime} / K^{\prime}} F i l_{\alpha} K_{2}^{\mathrm{top}} L^{\prime} \supset F i l_{\beta} K_{2}^{\mathrm{top}} K^{\prime}
$$

for any $\beta>\Phi_{L^{\prime} / K^{\prime}}(\alpha)$. Applying $N_{K^{\prime} / K}$ to the above formula, we obtain in view of Proposition 6.2:

$$
F i l_{\Phi_{L / K}(\alpha)} K_{2}^{\mathrm{top}} K \supset N_{L / K} F i l_{\alpha} K_{2}^{\mathrm{top}} L \supset F i l_{\beta} K_{2}^{\mathrm{top}} K
$$

for any $\beta>\Phi_{L / K}(\alpha)$. By class field theory, $K_{2}^{\text {top }} K^{\prime} / N_{L^{\prime} / K^{\prime}} K_{2}^{\text {top }} L^{\prime}$ is cyclic of prime degree. Therefore, $K_{2}^{\mathrm{top}} K^{\prime}$ is generated by $N_{L^{\prime} / K^{\prime}} K_{2}^{\mathrm{top}} L^{\prime}$ and $a$, where $a \notin$ $F i l_{\beta} K_{2}^{\text {top }} K^{\prime}$ for any $\beta>\Phi_{L / K}(\alpha)$. Therefore, $K_{2}^{\text {top }} K$ is generated by $N_{K^{\prime} / K} a$ and $N_{K^{\prime} / K} N_{L^{\prime} / K^{\prime}} K_{2}^{\mathrm{top}} L^{\prime}$. Since

$$
N_{K^{\prime} / K} a \in N_{K^{\prime} / K} F i l_{\Phi_{L / K}(\alpha)} K_{2}^{\mathrm{top}} K^{\prime}=F i l_{\Phi_{L / K}(\alpha)} K_{2}^{\mathrm{top}} K^{\prime}
$$

we conclude that $\left(F i l_{\Phi_{L / K}(\alpha)} K_{2}^{\mathrm{top}} K: N_{L / K} F i l_{\alpha} K_{2}^{\mathrm{top}} L\right)=[L: K]$.

2. The case, when $K$ is almost standard, and $h=-1$, i. e., $L / K$ is unramified. Then for $\alpha \geq(\mathbf{c}, \infty)$ the assertion follows directly from the definition of $F i l_{\alpha}$ with use of Proposition 0.4.4. The case of $\alpha=(\mathbf{c}, i)$ also follows immediately from the definition of $F i l_{\alpha}$. Further,

$$
\begin{aligned}
N_{L / K} F i l_{0} K_{2}^{\mathrm{top}} L & =N_{L / K}\left(U(1) K_{2}^{\mathrm{top}} L+\{t\} \cdot \mathcal{R}_{L}\right) \\
& \left.=N_{L / K} U(1) K_{2}^{\mathrm{top}} L+\{t\} \cdot N_{L / K} \mathcal{R}_{L}\right) \\
& =U(1) K_{2}^{\mathrm{top}} K+\{t\} \cdot \mathcal{R}_{K} .
\end{aligned}
$$

Finally, $N_{L / K} F i l_{-1} K_{2}^{\text {top }} L=N_{L / K} K_{2}^{\text {top }} L$ is a subgroup of index $[L: K]$ in $K_{2}^{\text {top }} K$ by class field theory.

The above argument also reduces Proposition to the case when both $K$ and $L$ are standard. In fact, since $K$ and $L$ are almost standard, there exists an unramified extension $K^{\prime} / K$ such that $K^{\prime}$ and $K^{\prime} L$ are standard. We can therefore identify $F i l_{\alpha} K_{2}^{\text {top }} K$ with $S_{\alpha, K}$, and $F i l_{\alpha} K_{2}^{\text {top }} L$ with $S_{\alpha, L}$.

3. Let $K$ be standard, $h=(\mathbf{c}, H), H>0$, or $h=H=0$. In both cases $L / K$ is a constant extension. Let $k$ and $l$ be the constant subfields of $K$ and $L$ respectively. Then $l / k$ is a cyclic extension with the jump of ramification $H$. By Proposition 
4.1.2, we obtain $N_{L / K} F i l_{\alpha} K_{2}^{\text {top }} L=F i l_{\alpha} K_{2}^{\text {top }} K$ for all $\alpha \geq(\mathbf{c}, \infty)$. Let $\alpha=(\mathbf{c}, i)$ or $\alpha=0=i$. Then

$$
N_{L / K} F i l_{\alpha} K_{2}^{\mathrm{top}} L=N_{L / K} T_{L}+N_{L / K}\left(\{t\} \cdot U_{i, l}\right)=T_{K}+\{t\} \cdot N_{L / K} U_{i, l} .
$$

It remains to notice that $N_{L / K} U_{i, l} \subset U_{\phi_{l / k}(i), k}$, these groups are equal if $i>H$, and $\left(U_{\phi_{l / k}(i), k}: N_{L / K} U_{i, l}\right)=[l: k]=[L: K]$ if $i \leq H$ because the last residue fields are quasi-finite.

4. In the remaining case $L / K$ is infernal. Since both $K$ and $L$ are standard, $L=k\{\{t\}\}, K=k\left\{\left\{N_{L / K} t\right\}\right\} . L / K$ is ferociously ramified, and Proposition 5.1.3 is applicable. This proves this case of Proposition for $\alpha \geq(\mathbf{c}, \infty)$. For $\alpha<(\mathbf{c}, \infty)$, notice that $N_{L / K}\left(\{t\} \cdot U_{i, k}\right)=\left\{N_{L / K} t\right\} \cdot U_{i, k}$.

\subsection{Main theorems.}

Theorem 1. Let $L / K$ be a finite abelian extension, $\alpha \in \mathbb{I}_{2}$. Then $N_{L / K} F i l_{\alpha} K_{2}^{\mathrm{top}} L$ is a subgroup in $\mathrm{Fil}_{\Phi_{L^{\prime K}(\alpha)}} K_{2}^{\mathrm{top}} K$ of index $\left|\operatorname{Gal}(L / K)_{\alpha}\right|$. Furthermore,

$$
F i l_{\Phi_{L / K}(\alpha)} K_{2}^{\mathrm{top}} K \cap N_{L / K} K_{2}^{\mathrm{top}} L=N_{L / K} F i l_{\alpha} K_{2}^{\mathrm{top}} L
$$

Proof. Use induction on $[L: K]$. If $[L: K]$ is prime, then the assertion of Theorem is just Proposition 6.3. In the general case let $h$ be the minimal ramification jump in $L / K, G^{\prime}=\cup_{\alpha>h} G_{\alpha}, G^{\prime \prime} \supset G^{\prime}$ such that $\left(G: G^{\prime}\right)$ is prime, $K^{\prime}=L^{G^{\prime}}$.

Let $\alpha>h$. By assumption of induction, $N_{L / K^{\prime}} F i l_{\alpha} K_{2}^{\text {top }} L$ is a subgroup in $F i l_{\Phi_{L / K^{\prime}}(\alpha)} K_{2}^{\text {top }} K^{\prime}$ of index $\left|\operatorname{Gal}\left(L / K^{\prime}\right)_{\alpha}\right|=\left|\operatorname{Gal}(L / K)_{\alpha}\right|$. Applying $N_{K^{\prime} / K}$, we obtain that $N_{L / K} F i l_{\alpha} K_{2}^{\text {top }} L$ is a subgroup of index $\leq\left|\operatorname{Gal}(L / K)_{\alpha}\right|$ in

$$
F i l_{\Phi_{K^{\prime} / K}\left(\Phi_{L / K^{\prime}}(\alpha)\right)} K_{2}^{\mathrm{top}} K=F i l_{\Phi_{L / K}(\alpha)} K_{2}^{\mathrm{top}} K .
$$

If this index is less than $\left|\operatorname{Gal}(L / K)_{\alpha}\right|$, we easily obtain that $\left(F i l_{\Phi_{L / K}(h)} K_{2}^{\mathrm{top}} K\right.$ : $\left.N_{L / K} F i l_{h} K_{2}^{\text {top }} L\right)<\left|\operatorname{Gal}(L / K)_{h}\right| \cdot\left[K^{\prime}: K\right]=[L: K]$. On the other hand, also by induction on $[L: K]$, the norm map induces an epimorphism of $K_{2}^{\text {top }} L / F i l_{h} K_{2}^{\text {top }} L$ onto $K_{2}^{\text {top }} K / F i l_{\Phi_{L / K}(h)} K_{2}^{\text {top }} K$, whence $\left(K_{2}^{\text {top }} K: N_{L / K} K_{2}^{\text {top }} L\right)<[L: K]$, a contradiction with class field theory.

In the case $\alpha \leq h$ we have $\left(F i l_{\Phi_{L / K}(\alpha)} K_{2}^{\mathrm{top}} K: N_{L / K} F i l_{\alpha} K_{2}^{\mathrm{top}} L\right) \geq[L: K]$ by class field theory, and

$$
\begin{aligned}
\left(F_{i} l_{\Phi_{L / K}(\alpha)} K_{2}^{\mathrm{top}} K: N_{L / K} F i l_{\alpha} K_{2}^{\mathrm{top}} L\right) \\
=\left(F i l_{\Phi_{L / K}(\alpha)} K_{2}^{\mathrm{top}} K: N_{K^{\prime} / K} F i l_{\Phi_{L / K^{\prime}}(\alpha)} K_{2}^{\mathrm{top}} K^{\prime}\right) \times \\
\quad \times\left(N_{K^{\prime} / K} F i l_{\Phi_{L / K^{\prime}}(\alpha)} K_{2}^{\mathrm{top}} K^{\prime}: N_{L / K} F i l_{\alpha} K_{2}^{\mathrm{top}} L\right) \\
\leq\left[K^{\prime}: K\right]\left[L: K^{\prime}\right]=[L: K] . \quad \square
\end{aligned}
$$

Theorem 2. Let $L / K$ be a finite abelian extension, $\Theta: K_{2}^{\text {top }} K / N_{L / K} K_{2}^{\text {top }} L \rightarrow$ $\operatorname{Gal}(L / K)$ the reciprocity map. Then

$$
\Theta\left(F i l_{\alpha} K_{2}^{\mathrm{top}} K \bmod N_{L / K} K_{2}^{\mathrm{top}} L\right)=\operatorname{Gal}(L / K)^{\alpha}
$$


for any $\alpha \in \mathbb{I}_{2}$.

Proof. Let $G=\operatorname{Gal}(L / K), \beta<\alpha, K^{\prime}=L^{G^{\alpha}}$. Then $\operatorname{Gal}\left(K^{\prime} / K\right)^{\alpha}$ is trivial, and $F i l_{\alpha} K_{2}^{\text {top }} K \subset N_{K^{\prime} / K} F i l_{\Psi_{K^{\prime} / K}(\alpha)} K_{2}^{\text {top }} K^{\prime}$ by Theorem 1. It follows

$$
\Theta\left(F i l_{\alpha} K_{2}^{\mathrm{top}} K \bmod N_{L / K} K_{2}^{\mathrm{top}} L\right) \subset \operatorname{Gal}\left(L / K^{\prime}\right)=\operatorname{Gal}(L / K)^{\alpha} .
$$

It remains to compare the indices of two subgroups by means of second assertion in Theorem 1.

\section{REFERENCES}

[A1] V. A. Abrashkin, Towards explicit description of ramification filtration in the 2-dimensional case, Preprint of Nottingham University 00-01 (2000), to appear in Proceedings of the conference "Ramification theory of arithmetic schemes" (Luminy, 1999).

http://www.maths.dur.ac.uk/pure/ps/va-drf.ps

[A2] , Ramification theory for higher dimenional local fields, Preprint of Durham University (2002).

http://www.maths.dur.ac.uk/pure/ps/va-mmud1.ps

[E] H. Epp, Eliminating wild ramification, Invent. Math. 19 (1973), 235-249.

[F1] I. B. Fesenko, Class field theory of multidimensional local fields of characteristic zero with residue field of positive characteristic, Algebra i Analiz 3 (1991), no. 3, 165-196; St. Petersburg Math. J. 3 (1992), 649-678.

[F2] Abelian local p-class field theory, Math. Ann. 301 (1995), 561-586.

[F3] Sequential topologies and quotients of Milnor K-groups of higher local fields, Algebra i analiz 13 (2001), 198-228; English translation to appear in St. Petersburg Math. J. 13 (2002), no. 3.

[FV] I. B. Fesenko, S. V. Vostokov, Local fields and their extensions: a constructive approach, AMS, Providence, 1993.

$[\mathrm{H}] \quad$ O. Hyodo, Wild ramification in the imperfect residue field case, Adv. Stud. Pure Math. 12 (1987), 287-314.

[HLF] I. B. Fesenko, M. Kurihara (eds.), Invitation to higher local fields, Geometry and Topology Monographs, vol. 3, 2000.

http://www.maths.warwick.ac.uk/gt/gtmcontents3.html

[Kn] B. Kahn, L'anneau de Milnor d'un corps local à corps residuel parfait, Ann. Inst. Fourier, Grenoble 34 (1984), no. 4, 19-65.

[KZ] M. V. Koroteev, I. B. Zhukov, Elimination of wild ramification, Algebra i Analiz 11 (1999), no. 6, 153-177; St. Petersburg Math. J. 11 (2000).

[L] V. G. Lomadze, On the ramification theory of two dimensional local fields, Mat. Sb. 109 (1979), no. 3, 378-394; Math. USSR-Sb. 37 (1980).

[MZ1] A. I. Madunts, I. B. Zhukov, Multidimensional complete fields: topology and other basic constructions, Trudy S.-Peterb. Mat. Obsch. 3 (1995), 4-46; Amer. Math. Soc. Transl. (Ser. 2) 165 (1995), 1-34.

[MZ2] _ Additive and multiplicative expansions in multidimensional local fields, Zapiski Nauchn. Sem. S.-Peterburg. Otdel. Mat. Inst. Steklov. (POMI) 272 (2000), 186-196.

[P] A. N. Parshin, Local class field theory, Trudy Mat. Inst. Steklov 165 (1984), 143-170; Proc. Steklov Inst. Math. 165 (1985), no. 3, 157-185.

[S] J.-P. Serre, Corps Locaux, 2nd ed., Hermann, Paris, 1968.

[Z1] I. B. Zhukov, Structure theorems for complete fields, Trudy S.-Peterb. Mat. Obschestva 3 (1995), 215-234; Amer. Math. Soc. Transl. (Ser. 2) 165 (1995), 175-192.

[Z2] Milnor and topological K-groups of multidimensional complete fields, Algebra $\mathrm{i}$ Analiz 9 (1997), no. 1, 98-147; St. Petersburg Math. J. 9 (1998), no. 1, 69-105.

The Department of Mathematics and Mechanics, St. Petersburg University, Bibliotechnaya pl. 2, St. Petersburg 198904, Russia.

E-mail: igor@zhukov.pdmi.ras.ru, igor_zh@hotmail.com 